\title{
Drawing Links from Transcriptome to Metabolites: The Evolution of Aroma in the Ripening Berry of Moscato Bianco (Vitis vinifera L.)
}

\author{
Laura Costantini $^{1 *}$, Christian D. Kappel ${ }^{2}$, Massimiliano Trenti $^{1}$, Juri Battilana ${ }^{1}$, \\ Francesco Emanuelli ${ }^{1}$, Maddalena Sordo ${ }^{1}$, Marco Moretto ${ }^{3}$, Céline Camps ${ }^{2}$, \\ Roberto Larcher ${ }^{4}$, Serge Delrot ${ }^{2}$ and Maria S. Grando ${ }^{1,5}$ \\ ${ }^{1}$ Grapevine Genetics and Breeding Unit, Genomics and Biology of Fruit Crop Department, Research and Innovation Centre, \\ Fondazione Edmund Mach, San Michele all'Adige, Italy, ${ }^{2}$ UMR Ecophysiology and Grape Functional Genomics, Institut des \\ Sciences de la Vigne et du Vin, University of Bordeaux, Villenave d'Ornon, France, ${ }^{3}$ Computational Biology Platform, \\ Research and Innovation Centre, Fondazione Edmund Mach, San Michele all'Adige, Italy, ${ }^{4}$ Experiment and Technological \\ Services Department, Technology Transfer Centre, Fondazione Edmund Mach, San Michele all'Adige, Italy, ${ }^{5}$ Center \\ Agriculture Food Environment, University of Trento, San Michele all'Adige, Italy
}

OPEN ACCESS

Edited by:

José Tomás Matus,

Centre for Research in Agricultural

Genomics, Spain

Reviewed by:

Philip Richard Young,

Institute for Wine Biotechnology,

South Africa

Patricio Hinrichsen,

Instituto de Investigaciones

Agropecuarias, Chile

*Correspondence:

Laura Costantin laura.costantini@fmach.it

Specialty section:

This article was submitted to Crop Science and Horticulture, a section of the journal

Frontiers in Plant Science

Received: 10 February 2017 Accepted: 25 April 2017

Published: 16 May 2017

Citation:

Costantini L, Kappel CD, Trenti M, Battilana J, Emanuelli F, Sordo M, Moretto M, Camps C, Larcher R,

Delrot S and Grando MS (2017) Drawing Links from Transcriptome to Metabolites: The Evolution of Aroma in the Ripening Berry of Moscato Bianco (Nitis vinifera L.).

Front. Plant Sci. 8:780. doi: 10.3389/fp/s.2017.00780
Monoterpenes confer typical floral notes to "Muscat" grapevine varieties and, to a lesser extent, to other aromatic non-Muscat varieties. Previous studies have led to the identification and functional characterization of some enzymes and genes in this pathway. However, the underlying genetic map is still far from being complete. For example, the specific steps of monoterpene metabolism and its regulation are largely unknown. With the aim of identifying new candidates for the missing links, we applied an integrative functional genomics approach based on the targeted metabolic and genome-wide transcript profiling of Moscato Bianco ripening berries. In particular, gas chromatography-mass spectrometry analysis of free and bound terpenoid compounds was combined with microarray analysis in the skins of berries collected at five developmental stages from pre-veraison to over-ripening. Differentially expressed metabolites and probes were identified in the pairwise comparison between time points by using the early stage as a reference. Metabolic and transcriptomic data were integrated through pairwise correlation and clustering approaches to discover genes linked with particular metabolites or groups of metabolites. These candidate transcripts were further checked for co-localization with quantitative trait loci (QTLS) affecting aromatic compounds. Our findings provide insights into the biological networks of grapevine secondary metabolism, both at the catalytic and regulatory levels. Examples include a nudix hydrolase as component of a terpene synthase-independent pathway for monoterpene biosynthesis, genes potentially involved in monoterpene metabolism (cytochrome P450 hydroxylases, epoxide hydrolases, glucosyltransferases), transport (vesicle-associated proteins, ABCG transporters, glutathione S-transferases, amino acid permeases), and transcriptional control (transcription factors of the ERF, MYB and NAC families, intermediates in light- and circadian cycle-mediated regulation with supporting evidence from the literature and additional regulatory genes with a previously unreported association to monoterpene accumulation).

Keywords: grapevine, Muscat, monoterpene, development, berry skin, metabolic and transcript profiling, integration, candidate gene 


\section{INTRODUCTION}

A great deal of the consumer interest in wine derives from its aroma characteristics. The major aroma-impact compounds in grape and wine are terpenoids (monoterpenes, sesquiterpenes, and in a wider acception also $\mathrm{C}_{13}$-norisoprenoids), phenylpropanoids/benzenoids, fatty acid derivatives, sulfur compounds, and methoxypyrazines (Dunlevy et al., 2009; Ebeler and Thorngate, 2009; Panighel and Flamini, 2014; Robinson et al., 2014; Black et al., 2015). The typical floral and citrus attributes of Muscat varieties are primarily determined by a combination of linalool, geraniol and nerol (Ribéreau-Gayon et al., 2000). The same monoterpenes contribute to the varietal aroma of Riesling in association with the linalool oxides, hydroxy-linalool, $\alpha$-terpineol, citronellol, terpendiol I and hydroxy-trienol (Rapp, 1998). Likewise rose oxide, which is highly correlated with Muscat score in grapes (Ruiz-García et al., 2014), is also a potent odorant in Scheurebe and Gewürztraminer (Guth, 1997; Ong and Acree, 1999; Luan et al., 2005).

The terpene biosynthetic pathway is generally well known (Dudareva et al., 2013), even though a number of alternative non-canonical reactions may occur (Sun et al., 2016). Of the two systems responsible for the production of plant isopentenyl diphosphate (IPP) and dimethylallyl diphosphate (DMAPP), the primarily cytosolic mevalonic acid (MVA) and the plastidial methylerythritol phosphate (MEP) pathway, the latter has been suggested as the dominant route for monoterpene biosynthesis in grape berries (Luan and Wüst, 2002). Several lines of evidence (Battilana et al., 2009, 2011; Duchêne et al., 2009; Emanuelli et al., 2010; Martin et al., 2012; Wen et al., 2015) support the existence of at least two rate-limiting enzymes in the grapevine MEP pathway, namely the first (1-deoxy-Dxylulose 5-phosphate synthase, VvDXS1) and the last (4-hydroxy3-methylbut-2-enyl diphosphate reductase, VvHDR). Both IPP and DMAPP are substrates for short-chain prenyltransferases, which produce prenyl diphosphate precursors for the large family of terpene synthases (TPSs). To date around 40 fulllength VvTPSs out of 53-89 predicted functional enzymes have been biochemically characterized (Martin et al., 2010) and some major players in grape Muscat aroma have been identified, like the $\alpha$-terpineol synthase $V v T e r$, the linalool synthase Lis, the linalool/nerolidol synthase VvPNLinNer1 and the geraniol synthase VvPNGer (Ebang-Oke et al., 2003; Martin and Bohlmann, 2004; Martin et al., 2012; Matarese et al., 2013; Zhu et al., 2014; Wen et al., 2015). Once a terpenoid alcohol skeleton has been produced, extensive modifications determine the final monoterpene composition of grapes and wines (Ribéreau-Gayon et al., 1975; Williams et al., 1989; Luan et al., 2004, 2005, 2006a,b; Mathieu et al., 2009). These secondary transformations are (at least in part) catalyzed by enzymes (Luan et al., 2006a; D'Onofrio et al., 2016) that in most cases have not been identified. The only exceptions are the three grape monoterpenol $\beta$-D-glucosyltransferases VvGT7, VvGT14 and VvGT15 and the cytochrome P450 CYP76F14 (Bönisch et al., 2014a,b; Ilc et al., 2017). The main reason for this gap is that such enzymes belong to large families with broad substrate tolerance and overlapping activities (Schwab, 2003; Nelson et al.,
2008; Schwab and Wüst, 2015). A better knowledge of the missing enzymes might allow us to manipulate the formation of grape aroma compounds. For example, limiting the reactions responsible for the depletion of key odorants (e.g., through the selection of genotypes with low monoterpene glycosyltransferase or oxygenase activities in breeding programs) could be an alternative approach for the improvement of grape/wine flavor (Bönisch et al., 2014a; Hjelmeland and Ebeler, 2015).

The grapevine terpenoid pathway is intricately regulated by endogenous and environmental factors that enable spatially and temporally controlled metabolite production (Ebeler and Thorngate, 2009; Robinson et al., 2014). In other plant species a network of transcription factors (TFs) is involved in the regulation of this pathway, including members of the AP2, AP2/ERF, bZIP, MYB, MYC, NAC, WRKY, and YABBY families (De Geyter et al., 2012; Patra et al., 2013; Nieuwenhuizen et al., 2015; Wang et al., 2016). A tight regulation of terpene biosynthesis is additionally exerted at the post-transcriptional level involving both structural enzymes and transcription factors (Vom Endt et al., 2002; Hemmerlin, 2013; RodríguezConcepción and Boronat, 2015), as observed also in Vitis vinifera (Bönisch et al., 2014a; Matarese et al., 2014). A number of transcription factors that might control terpene synthesis have been recently predicted in grapevine through gene co-expression network analysis (Wen et al., 2015), though none of them has been yet demonstrated to regulate the expression of relevant terpene pathway genes. Similarly, the reasons of the differential accumulation of the main monoterpenes in grape berry tissues across development (Günata et al., 1985; Wilson et al., 1986; Park et al., 1991; Luan and Wüst, 2002), which is reflected in the identification of specific QTLs for linalool and geraniol/nerol (Doligez et al., 2006; Battilana et al., 2009), are still unknown.

This work aims at a better understanding of aroma determination in grapevine and at the identification of candidate genes for further functional analysis. To this purpose, we integrated gas chromatography/mass spectrometrybased quantitative analysis of selected metabolites with microarray-based transcriptomic analysis in Moscato Bianco (Vitis vinifera L.) ripening berries. According to the observed associations between metabolite and transcript profiles, we report several genes that may control the accumulation of free and glycosidically bound monoterpenes and additional aroma-related compounds.

\section{MATERIALS AND METHODS}

\section{Plant Material For Metabolic and Microarray Analyses}

Berries of the cultivar Moscato Bianco (Vitis vinifera L.) were collected from pre-veraison to over-ripening in 2005, 2006, and 2007 (Figure 1A and Table 1). At each sampling date, ten bunches were taken from ten adult plants out of the $\sim 250$ grown on Kober 5BB rootstocks in the experimental fields of FEM (Fondazione Edmund Mach, San Michele all'Adige, Italy). Care was taken to sample from different vines and positions within each vine. In the lab, berries were pooled in order 


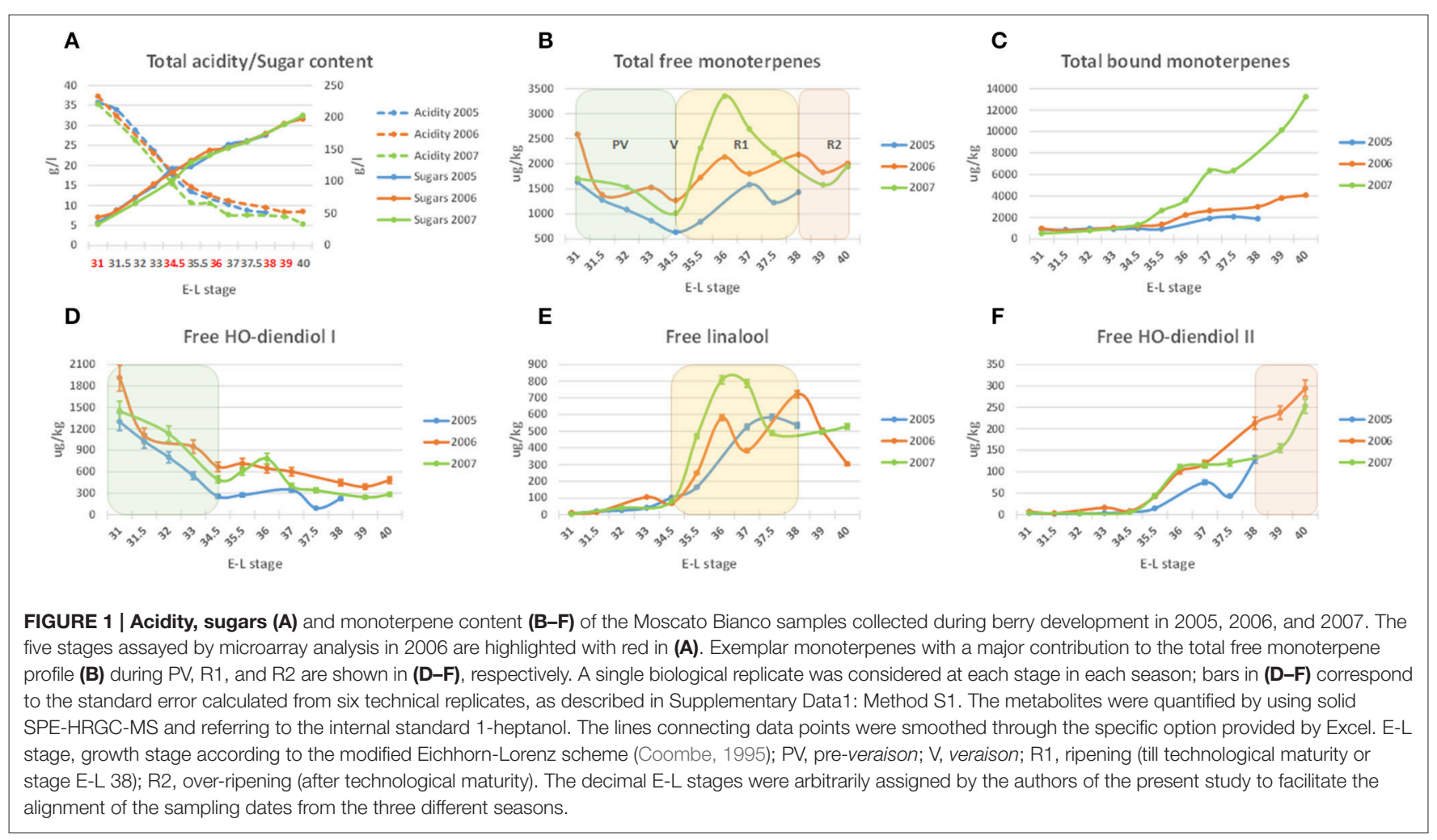

TABLE 1 | Acidity and sugar content of the Moscato Bianco samples collected during berry development in 2006.

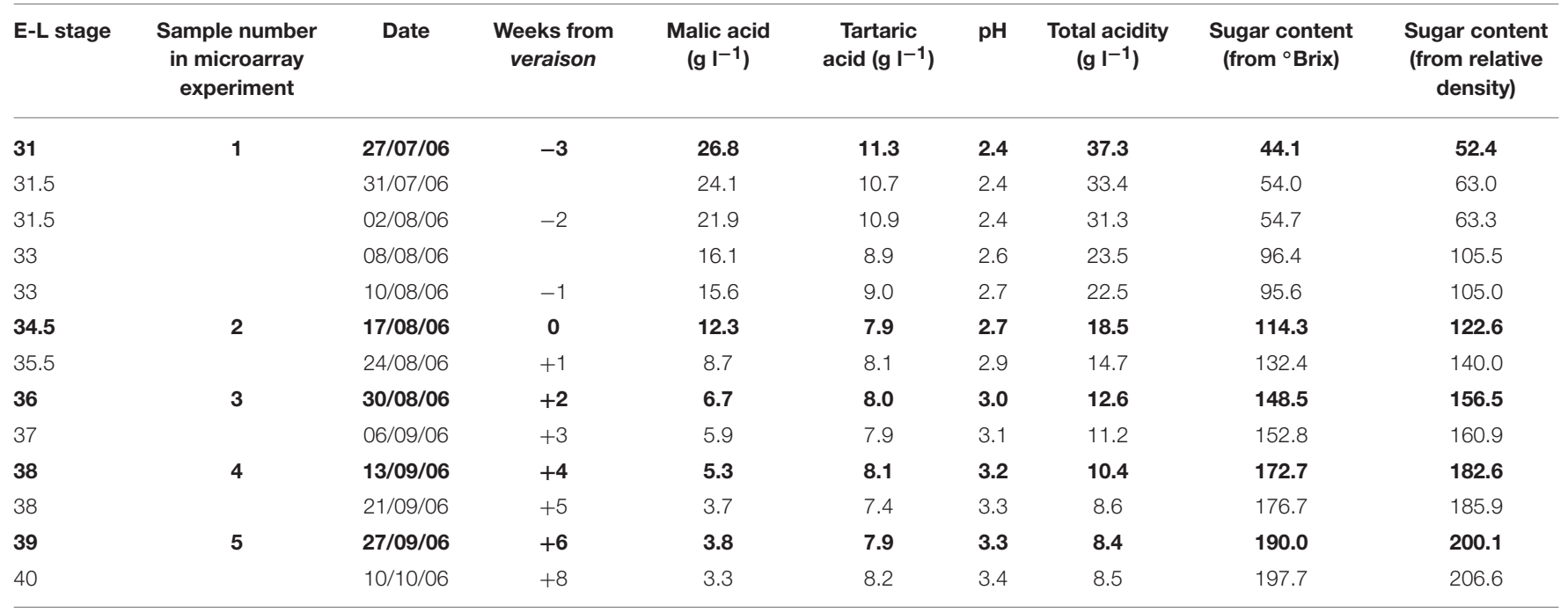

The five stages used for microarray analysis are in boldface. E-L stage, growth stage according to the modified Eichhorn-Lorenz scheme (Coombe, 1995). The decimal E-L stages were arbitrarily assigned by the authors of the present study to facilitate the alignment of sampling dates from three different seasons (Figure 1). The weeks from veraison were established with a maximum tolerance of 2 days from the exact date.

to minimize environmental effects and then divided into two (2005 and 2007) or three (2006) batches. Berries from the first batch were homogenized to juice $(80 \mathrm{~mL})$ and analyzed for titratable acidity and soluble solids content by FT-IR (Fourier Transform InfraRed) spectroscopy with a FOSS instrument (FOSS NIRSystems, Oatley, Australia). Berries from the second batch were stored at $-80^{\circ} \mathrm{C}$ till metabolic analysis. Berries from the third batch were hand-peeled, the skins were immediately frozen in liquid nitrogen and stored at $-80^{\circ} \mathrm{C}$ pending RNA extraction.

\section{Metabolic Analysis}

Thirty-two aroma-active compounds were quantified in their free and glycosidically bound form by using solid phase 
extraction (SPE) and high-resolution gas chromatography-mass spectrometry (HRGC-MS; Supplementary Data1: Method S1 and Supplementary Table S1) in the growing seasons 2005, 2006, and 2007.

Network analysis for 2006 metabolic data included pairwise correlation, hierarchical clustering with bootstrapping (Pvclust with 10,000 resamplings, see Suzuki and Shimodaira, 2006) and principal component analysis (PCA) and was applied to different data sets (free and glycosidically bound metabolites, absolute quantities and differentials, 5 and 13 time points).

\section{Microarray Analysis}

Based on monoterpene accumulation during berry development in 2006 (Figure 2), five time points were chosen along this season (Figure 1 and Table 1). Total RNA was extracted from grape skins using the Spectrum ${ }^{\mathrm{TM}}$ Plant Total RNA Kit (SigmaAldrich, St. Louis, Missouri, USA). RNA quantity and quality were evaluated with a NanoDrop ND-8000 spectrophotometer
(NanoDrop Technologies, Wilmington, Delaware, USA) and an Agilent 2100 Bioanalyzer (Agilent Technologies, Mississauga, Ontario, Canada).

Microarray experiments were carried out with a 70-mer oligoarray containing all 14,562 probes from the ArrayReady Oligo Set ${ }^{\mathrm{TM}}$ (AROS) for the Grape (Vitis vinifera) Genome version 1.0 (Operon Biotechnologies, Huntsville, Alabama, USA). At the time this platform represented a good compromise between genome coverage, cost and computational effort required for data analysis. RNA from points 2 to 5 was hybridized competitively with RNA from point 1 (pre-veraison), following the dye-swap experiment design (Churchill, 2002). A total of sixteen slides were used (four comparisons: 2 vs. 1,3 vs. 1,4 vs. 1,5 vs. 1 ; two biological and two technical replicates). The biological and technical replicates corresponded to two subgroups from the unique pool of berries (third batch) and to the dye swaps, respectively. Details for probe synthesis,

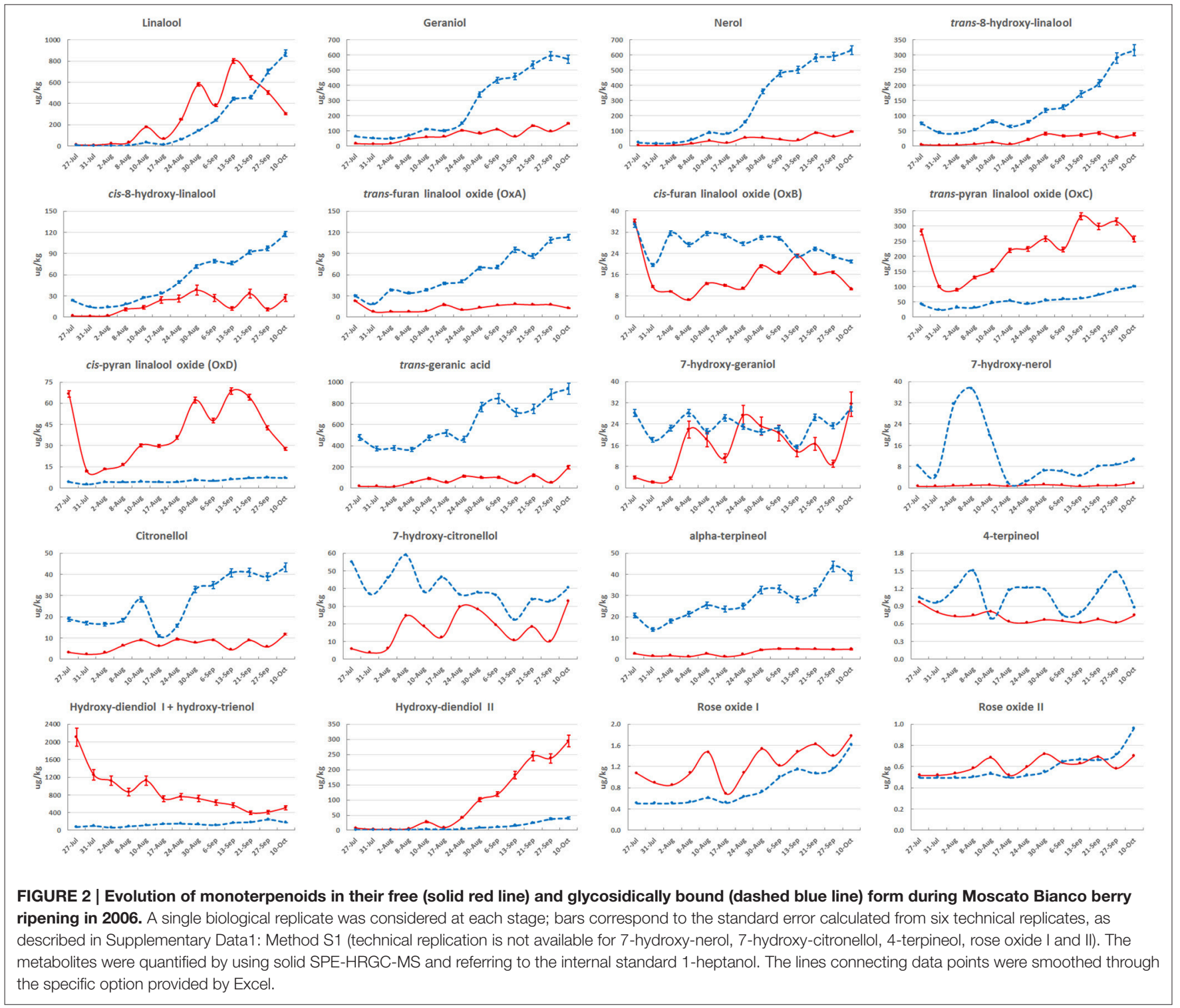


hybridization and scanning are described in Supplementary Data1: Method S2.

Spot intensities were quantified with the software MAIA 2.75 (Novikov and Barillot, 2007). After excluding poor quality spots due to bad spotting (e.g., spots with irregular shapes or highly unequal intensity distributions), median intensity gene expression data without background subtraction were normalized by a global lowess method followed by a print-tip median method with a modified version of the Goulphar script version 1.1.2 (Lemoine et al., 2006). Differentially expressed probes (DEPs) with a false discovery rate (FDR) $<1 \%$ and a cutoff of 2-fold change (FC) were identified with the R/Bioconductor Limma package using linear models (Smyth, 2004) and taking into account biological and technical replicates by doing a twofactor analysis. The earliest sample was used as the reference to whom all the other samples were compared. A multiple testing correction (Benjamini and Hochberg, 1995) was applied to adjust the FDR. The full raw expression dataset is available at the Gene Expression Omnibus (GEO, http://www.ncbi.nlm.nih.gov/geo/) under the accession number GSE76834.

\section{Probe Functional Annotation}

The 70-mer probes spotted on the Grape AROS V1.0 array represent 14,562 transcripts from The Institute for Genomic Research (TIGR) Grape Gene Index (VvGI), release 3 (August 13, 2003). The corresponding annotation is based upon a match between each oligo and the gene set of the $12 \mathrm{X}$ version of the grape genome at CRIBI (http://genomes.cribi.unipd.it/grape/) and is publicly available at GEO (http://www.ncbi.nlm.nih.gov/geo/) under the accession number GPL15453. Since this annotation provides every oligo with a text description but doesn't associate it to any gene prediction identifier, we independently achieved this information by blastN alignment against the grape gene sets at CRIBI (http://genomes.cribi.unipd.it/grape/, 12X version of the genome, V1 gene prediction, annotation from Grimplet et al., 2012) and IASMA (Velasco et al., 2007), as fully detailed in Supplementary Table S2. For the alignment against the CRIBI gene set, the following parameters were used: sequence identity $\geq 90 \%$, minimum alignment length of $95 \%$, maximum number of mismatches of 5 and maximum number of gaps of 5 . The aligned 70-mers were found to correspond to 7,162 and 8,260 unique gene predictions at CRIBI and IASMA, respectively. The 14,562 probes were also grouped into main functional categories according to the Mapman BIN structure (Rotter et al., 2009; Supplementary Table S2).

\section{Microarray Validation via Real-Time PCR}

Real-time quantitative reverse transcription-PCR (qRT-PCR) was used to validate the microarray results. Since RNA from berries collected in 2006 was no more available, new samples (with three biological replicates from pooled berries) as closest as possible to those analyzed in 2006 were obtained in 2016 by adopting the same sampling procedure and the same protocol for RNA extraction.

Primers for the amplification of unique PCR products from 70 to $250 \mathrm{bp}$ were designed on 15 Vitis vinifera gene predictions perfectly matching with the microarray 70 -mers by using Primer3 (Untergasser et al., 2012), as reported in Supplementary Table S3. Details for the amplification reaction and expression analysis are described in Supplementary Data1: Method S3. The relationship between microarray and qRT-PCR data was established through Pearson correlation.

\section{Discovery of a Link between Transcriptome and Metabolome in Aroma Development Integration of 2006 Transcriptomic and Metabolic Data}

Different approaches were tested in order to discover transcripts linked with the accumulation of one or more metabolites. In a first identification step of candidates, the most stable expression changes were preferred to the biggest ones, hence the microarray probes with adjusted $p$-values $<0.05$ in all comparisons were considered, irrespective of their fold change $(4,450)$. Working at probe level instead of gene level was chosen for two main reasons: (1) different probes supposedly matching to the same gene (especially long genes) often show different expression values, which might be an indicator of alternative transcription and (2) the sequence specificity to CRIBI $12 \mathrm{X}$ gene predictions is not optimal for a number of probes spotted on the AROS array (this is especially true for probes related to secondary metabolism); for a detailed assessment of probe specificity, Moscato Bianco genome and transcriptome assembly would be required, which is out of the scope of this work.

\section{Pairwise correlation}

Pearson pairwise correlation was calculated between transcripts and metabolites across all the time points $\left(\log _{2}\right.$-transformed differentials in the pairwise comparisons 2 vs. 1,3 vs. 1,4 vs. 1 , and 5 vs. 1). With the goal of identifying aroma regulatory genes we also tested a two-step strategy, which was based on (1) search for candidate metabolism and transport genes by direct correlation with metabolites and (2) expression pairwise correlations between these enzyme/transporter-coding genes and any regulatory gene within the microarray. For this aim, Pearson correlations were computed both between differential gene expression ratios $(n=4)$ and microarray channel intensities $(n$ $=32$, when considering technical replicates separately).

\section{Correlation biclustering}

Based on the assumption that a gene might regulate the accumulation of a metabolite only at specific stages during ripening, correlation biclustering between transcripts and metabolites was achieved with QUBIC (Li et al., 2009) ( $\log _{2}-$ transformed data, Pearson correlation). Compared to the traditional clustering methods, biclustering algorithms discover local co-expression patterns (groups of genes/metabolites that show similar patterns under a specific subset of the experimental conditions) (Madeira and Oliveira, 2004). We manually inspected our biclustering data only in a few exemplar cases.

\section{Soft clustering}

Soft clustering of the metabolite and transcript differentials was performed by using the R/Bioconductor Mfuzz package (Kumar 
and Futschik, 2007) with the default value 1.25 for the fuzzy parameter $\mathrm{m}$. A membership value in the range of $0-1$ was assigned to each metabolite and probe. Soft clustering offers several advantages with respect to hard clustering; in particular, it has been suggested to be more suitable for time course microarray data in which expression patterns are often not well separated (Futschik and Carlisle, 2005; Kumar and Futschik, 2007). The biological significance of the clusters was analyzed by enrichment analysis of the MapMan functional categories assigned to the probes in each cluster. Specifically, Chi square and Fisher statistical tests were employed to search for significant differences ( $p$-value $<0.05$ ) between the observed number of probes within each MapMan functional category per cluster and the expected number of probes in that category based on the overall AROS genome array expression distribution.

\section{Selection of candidate genes}

From the whole set of transcripts with a potential association to monoterpenes (based on their correlation with metabolites and membership to soft clusters/biclusters harboring metabolites) we selected a subset of genes with significant expression changes and/or supporting evidence from the literature, like a relevant function in other plant species, co-localization with QTLs for monoterpene content and coexpression with genes involved in the terpene pathway. In particular, the QTL co-localization was stated when the V1 gene predictions fell into the 1-LOD confidence intervals of the QTLs for linalool, geraniol and nerol reported by Doligez et al. (2006) and Battilana et al. (2009) based on the analysis of different segregating progenies in 23 seasons (depending on the progeny). The genomic region corresponding to each QTL confidence interval was determined from the physical position of the two neighboring markers, while the V1 gene prediction physical position was retrieved from Grimplet et al. (2012).

\section{Integration of Transcriptomic and Metabolic Data Over Multiple Seasons to Verify a Subset of Candidate Genes}

For the candidate genes assessed by both microarray and real-time PCR analyses (in 2006 and 2016, respectively), the association between expression and metabolic profiles was further tested by employing a general monoterpene quantification that considers the three seasons $(2005,2006$, and 2007 ) as replicates. To this purpose, the average concentration among these seasons was computed for each metabolite at each developmental stage. Pearson and Spearman correlations were calculated between the transcriptional and metabolic data expressed as $\log _{2}$ fold changes at the stages $2-5$ (E-L 34.5, 36, 38, and 39) with respect to the first stage (E-L 31).

\section{RESULTS AND DISCUSSION}

Our study gives an example of the systems biology approach. Systems biology has been successfully applied to the discovery of regulatory and biosynthetic genes involved in the control of metabolite production (Yuan et al., 2008; Liberman et al., 2012), including examples from grape (Zamboni et al., 2010; Fortes et al., 2011; Agudelo-Romero et al., 2013; Costantini et al., 2015; Malacarne et al., 2015; Suzuki et al., 2015; Wen et al., 2015; Savoi et al., 2016).

\section{Metabolic Analysis}

The present work provides a temporal profiling of aromatic compounds in the Moscato Bianco ripening berry. The protocol used for the chemical analysis was optimized for molecules belonging to the monoterpenoid class, however it allowed the simultaneous quantification of additional metabolites. In particular, the content of 21 monoterpenoids, $3 \mathrm{C}_{13^{-}}$ norisoprenoids, 5 phenylpropanoids/benzenoids and $3 \mathrm{C}_{6}$ aliphatic compounds was quantified from pre-veraison to overripening in 2005, 2006, and 2007. For several compounds a coherent accumulation trend was observed in the different years (Figure 1 and Supplementary Figure S1A). The most significant correlation between seasons was observed for free linalool, nerol, $\alpha$-terpineol, hydroxy-diendiol I + hydroxy-trienol, hydroxydiendiol II, hexanol, cis-3-hexen-1-ol, bound linalool, geraniol, nerol, trans-furan linalool oxide and benzyl alcohol. Other metabolites appeared instead to be more sensitive to seasonal effects, like rainfall and temperature (Supplementary Figure S1B). Hereafter, we will refer to 2006, which is the year assayed by microarray analysis.

The most abundant metabolites were monoterpenes (hydroxy-diendiol I, trans-geranic acid, linalool, geraniol and nerol), with concentrations higher than $600 \mu \mathrm{g} / \mathrm{kg}$ of berries (Figure 2 and Supplementary Figure S2). The majority of monoterpenoids reached the highest amount in their glycosidically bound form. The main exceptions are represented by high oxidation state monoterpenes, like the two pyran linalool oxides, the two diendiols and rose oxide I. A clear prevalence of the free form was also observed for the $\mathrm{C}_{6}$ aliphatic compounds, while the most abundant $\mathrm{C}_{13}$-norisoprenoids and phenylpropanoids/benzenoids were glycosidically bound, in agreement with previous analyses (Sánchez Palomo et al., 2006; D’Onofrio et al., 2016).

The quantity of many metabolites was significantly (at least 2 -fold) altered during ripening. The compounds that changed most with respect to pre-veraison were linalool, geraniol, nerol, cis/trans-8-hydroxy-linalool, hydroxy-diendiol I and II in both forms; cis-furan linalool oxide, trans-geranic acid, 7hydroxy-geraniol, 7-hydroxy-citronellol, hydroxy-trienol in their free form; trans-furan linalool oxide, 3-oxo- $\alpha$-ionol, methyl salicylate, hexanol and cis-3-hexen-1-ol in their bound form (Supplementary Figures S3A,D, Supplementary Table S4).

The pattern of accumulation along berry development varied with the metabolite (Figure 2 and Supplementary Figure S2). The concentration of the three compounds mainly contributing to Muscat aroma (linalool, geraniol, and nerol) was from low to moderate before veraison (August 17 or stage E-L 34.5 in this work) and then increased during ripening. Free linalool reached its maximum on September 13 (technological maturity or stage E-L 38) and decreased during over-ripening. A similar behavior was observed in 2005 and 2007, even though the peak corresponded to slightly earlier stages (Figure 1E), proving that technological and aroma ripening might not occur at the same 
time (Vilanova et al., 2012). Otherwise, free geraniol and nerol as well as the three bound forms showed a steady increase in their content during the sampling period. These results confirm previous findings (Günata et al., 1985; Ebang-Oke et al., 2003; Piazzolla et al., 2016). Several additional patterns were observed. For example, the four linalool oxides could be detected at berry onset; their concentration reached a minimum between July 31 (stage E-L 31.5 in this work) and August 8 (stage E-L 33) and then increased in at least one of the two forms to peak on September 13 (stage E-L 38) in their free form. While the glycosidically bound forms of the two diendiols showed a similar pattern of accumulation, free hydroxy-diendiol I and hydroxytrienol were highly concentrated before veraison and decreased over the course of berry ripening, with a trend opposite to that of free hydroxy-diendiol II (Figures 1, 2). The high content of free hydroxy-diendiol I and hydroxy-trienol at berry onset, when free linalool was not yet produced, may indicate that their accumulation is regulated independently from that of their precursor.

In the attempt of simplification, metabolite network analysis was performed on a total of 52 (26 free and 26 glycosidically bound) compounds. Metabolite grouping was obtained through hierarchical clustering and principal component analysis by using different metabolic data sets (Figure 3 and Supplementary Figures S3, S4). It is clearly evident that most monoterpenes are tightly correlated, which is indicative of their common metabolic origin and in agreement with previous findings (Ilc et al., 2016b). In particular, when considering the absolute amount of free metabolites at 13 time points, three main clusters (AU > 95\%) could be identified: (1) cis-pyran linalool oxide (OxD), transpyran linalool oxide $(\mathrm{OxC})$, trans-furan linalool oxide (OxA) and cis-furan linalool oxide (OxB); (2) geraniol, nerol, cis-8hydroxy-linalool, benzyl alcohol, 2-phenylethanol, trans-geranic acid, citronellol, hydroxy-diendiol II, trans-8-hydroxy-linalool, linalool, hexanol, trans-3-hexen-1-ol, rose oxide I, rose oxide II and $\alpha$-terpineol; (3) 4-terpineol and hydroxy-diendiol I + hydroxy-trienol. Additionally, within the second cluster a clear separation could be noticed between linalool on one side, geraniol and nerol on the other side (Figure $\mathbf{3 A}$ and Supplementary Figure S4A). Oppositely, when considering the absolute amount of bound metabolites at 13 time points a single significant cluster was obtained, which included most of the analyzed compounds. It can be easily noticed that bound cis-furan linalool oxide $(\mathrm{OxB})$ has a peculiar behavior with respect to the other three linalool oxides (Figure 3B and Supplementary Figure S4C).

\section{Microarray Analysis}

Gene expression in Moscato Bianco berry skin at stages 2-5 was compared to stage 1 (pre-veraison). The two biological replicates assayed at each stage were confirmed to perfectly cluster together (Supplementary Figure S5). The total number of differentially expressed probes (DEPs) in at least one comparison was 2,228, which corresponds to $15 \%$ of the chip probes (Supplementary Table S5). As expected, the highest number of DEPs was observed in stage 5 vs. stage 1 (616 up-regulated and 1,132 down-regulated probes), whereas the lowest number was recorded in stage 2 vs. stage 1 (452 up-regulated and 506 down-regulated probes).
A number of DEPs were common among comparisons (21, 19, and $28 \%$ of common DEPs among 2, 3, and 4 comparisons, respectively), whereas $32 \%$ of the DEPs were regulated at only one time point (data calculated from Supplementary Table S5).

\section{Microarray Validation via Real-Time PCR}

Specific primers were designed for 15 candidate genes and the change in their expression during berry development was analyzed in skin tissues by qRT-PCR to validate the microarray dataset (Figure 4). A strong relationship was found between the microarray and qPCR fold changes in the expression levels of the 15 genes (overall Pearson correlation coefficient $r=0.84$, with individual values ranging from 0.47 to 1 ), indicating the reliability of the whole transcriptome assay (Figure 4 and Supplementary Table S3).

\section{Discovery of a Link between Transcriptome and Metabolome in Aroma Development}

Potential links between transcripts and metabolites were established based on correlation and clustering approaches, though they do not necessarily imply causation. To this purpose, 4,450 probes with adjusted $p$-values $<0.05$ in all comparisons were considered, which included 1,906 out of the 2,228 DEPs with a cut-off of 2 fold-change and adjusted $p$-value $<0.01$.

\section{Integration of 2006 Transcriptomic and Metabolic Data}

\section{Pairwise correlation}

Significant (at the 0.05 or 0.01 level) Pearson correlations could be established only in the absence of Benjamini and Hochberg (1995) correction for multiple testing (Supplementary Table S6). Consequently, this result was employed as criterium in the following candidate gene selection only in combination with additional supporting evidence. In the two-step strategy, positive pairwise expression correlations were discovered between 33 enzyme/transporter-coding genes correlated to metabolites and several regulatory genes within the microarray (Supplementary Table S6).

\section{Correlation biclustering}

Correlation clustering between transcripts and metabolites resulted in the identification of 419 biclusters, that are groups of probes with a common behavior toward a group of metabolites. The clustered probes and metabolites were found to belong to a number of biclusters ranging from 1 to 10 and from 3 to 182, respectively (Supplementary Table S7).

\section{Soft clustering}

Based on their expression profile across stages 1-5, the selected 4,450 probes and 52 metabolites were clustered into nine distinct Mfuzz groups (Figure 5 and Supplementary Table S8). The distribution of probes per cluster within each MapMan functional category is shown in Supplementary Figure S6 and the enriched categories within each cluster are indicated in Figure 5. Probes annotated with the Mapman functional category "Secondary metabolism" were not found to be significantly over-represented in any cluster. Free geraniol and nerol were attributed to a distinct 

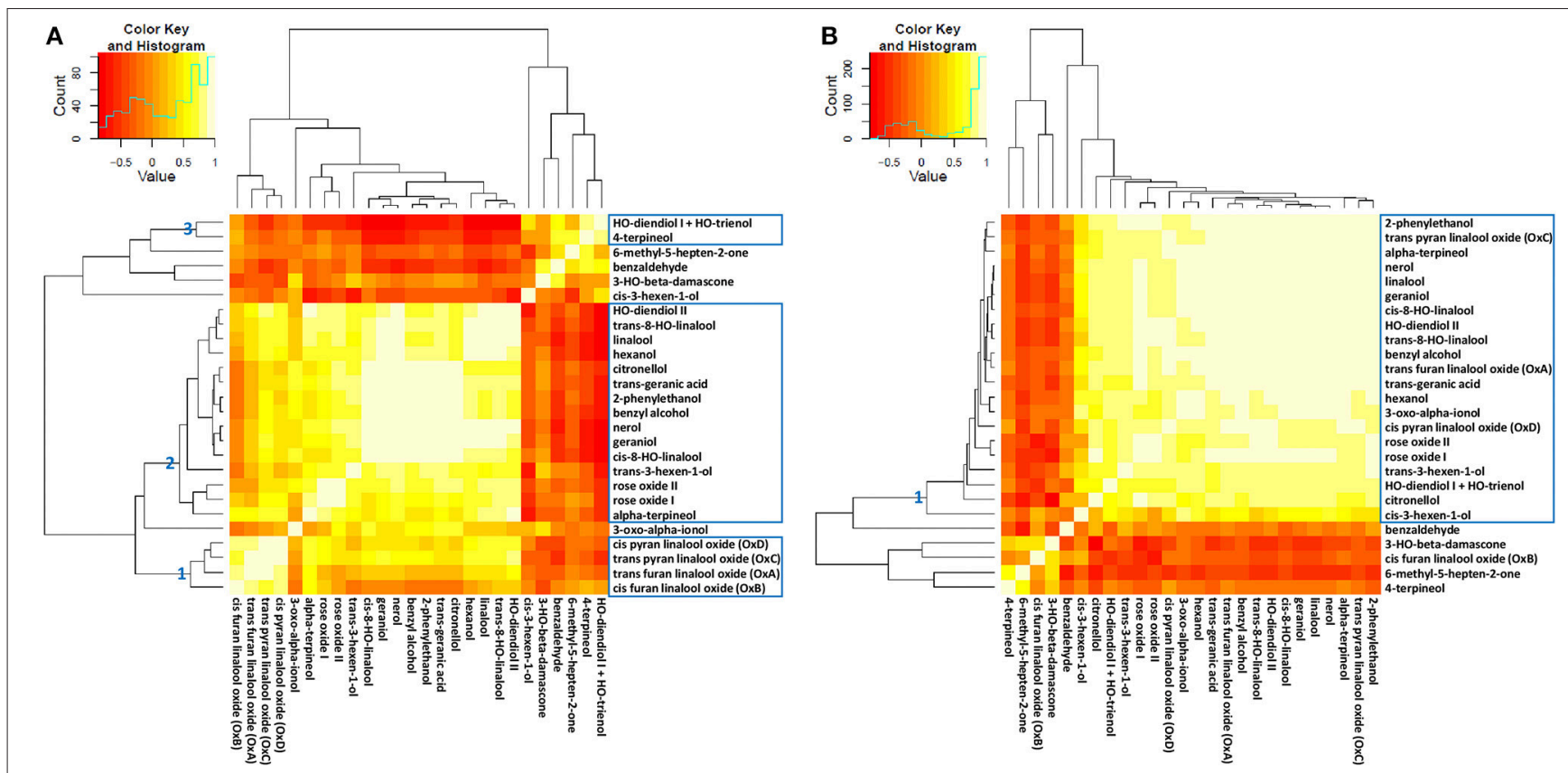

FIGURE 3 | Correlation heatmap of free (A) and bound (B) $\log _{2}$-transformed metabolites analyzed at 13 time points in 2006. The dendrograms are the output of Pvclust clustering (for details see Supplementary Figures S4A,C). The intensity of yellow coloration indicates the strength of relationships between metabolites.

cluster (cluster 6) with respect to free linalool (cluster 9), which reflects their Pvclust clustering (Figure 3A and Supplementary Figure S4A). This separation is mainly due to the decrease of free linalool, but not geraniol and nerol, from technological maturity onwards (Figure 2). The highly similar accumulation trend of geraniol and nerol likely reflects a common chemical origin (nerol is a geometrical isomer of geraniol), while their relationship with linalool is less clear. Oppositely, the bound forms of the three monoterpenoids accumulated to a similar extent (cluster 4 in Figure 5), suggesting dynamic changes in the distribution and concentration of these compounds.

\section{Selection of Candidate Genes}

Several genes with a potential association to aroma-related compounds were identified from the probes correlated and clustered with those metabolites (Supplementary Table S9, Supplementary Discussion in Supplementary Datal and Supplementary Table S10). In particular, the contrasting behavior of free linalool and free geraniol/nerol encouraged us to search for genes specifically related to one or the other profile. The existence of linalool-specific metabolic pathways is even more intriguing if we consider that the prevalence of the linalool class on the geraniol one clearly distinguishes Moscato Bianco from other aromatic varieties (D'Onofrio et al., 2016).

From this broad gene set, the most promising candidates for monoterpene biosynthesis and its regulation were further selected (Table 2) based on supporting evidence from the literature, e.g., a relevant role for the homolog gene in other plant species, the co-localization with QTLs for monoterpene content (with a special attention to the linalool-specific QTLs on chromosomes 2 and 10, which were also detected in the
Moscato Bianco genetic background by Battilana et al., 2009), or the coexpression from public transcriptomic databases with genes involved in the metabolic pahways under study, which may indicate functional association according to the "guilt-byassociation" principle. Hereafter, we discuss the most interesting findings from the present work; obvioulsy, we can't exclude that additional genes not included in this microarray platform may participate in monoterpene biosynthesis, as well as we can't know a priori whether our findings will be reproduced in other Muscat varieties with a genetic background different from Moscato Bianco.

\section{Monoterpene skeleton biosynthesis}

Early terpenoid pathway genes. The role of VvDXS isoforms in the development of aroma was previously investigated by realtime PCR on the same samples of Moscato Bianco analyzed here (Battilana, 2009), for which reason we did not repeat the analysis. In that study a significant up-regulation of $V v D X S 1$ was found to precede the peak of linalool, geraniol and nerol resulting in a positive correlation between $V v D X S 1$ expression profile and monoterpenoid accumulation. On the Grape AROS V1.0 array no probe could be found for $V v D X S 1$, whereas four probes corresponding to other DXS isoforms (VIT_04s0008g04970 and VIT_00s0218g00110) were not differentially expressed during Moscato Bianco berry ripening.

Several pieces of evidence from different plant species suggest that flux control in the MEP pathway does not converge on a single rate-limiting enzyme, such as DXS, but may involve other enzymes like DXR (1-deoxy-D-xylulose 5-phosphate reductoisomerase) and HDR (Vranová et al., 2012; Hemmerlin, 2013). The lack of significant modulation and the decreasing 


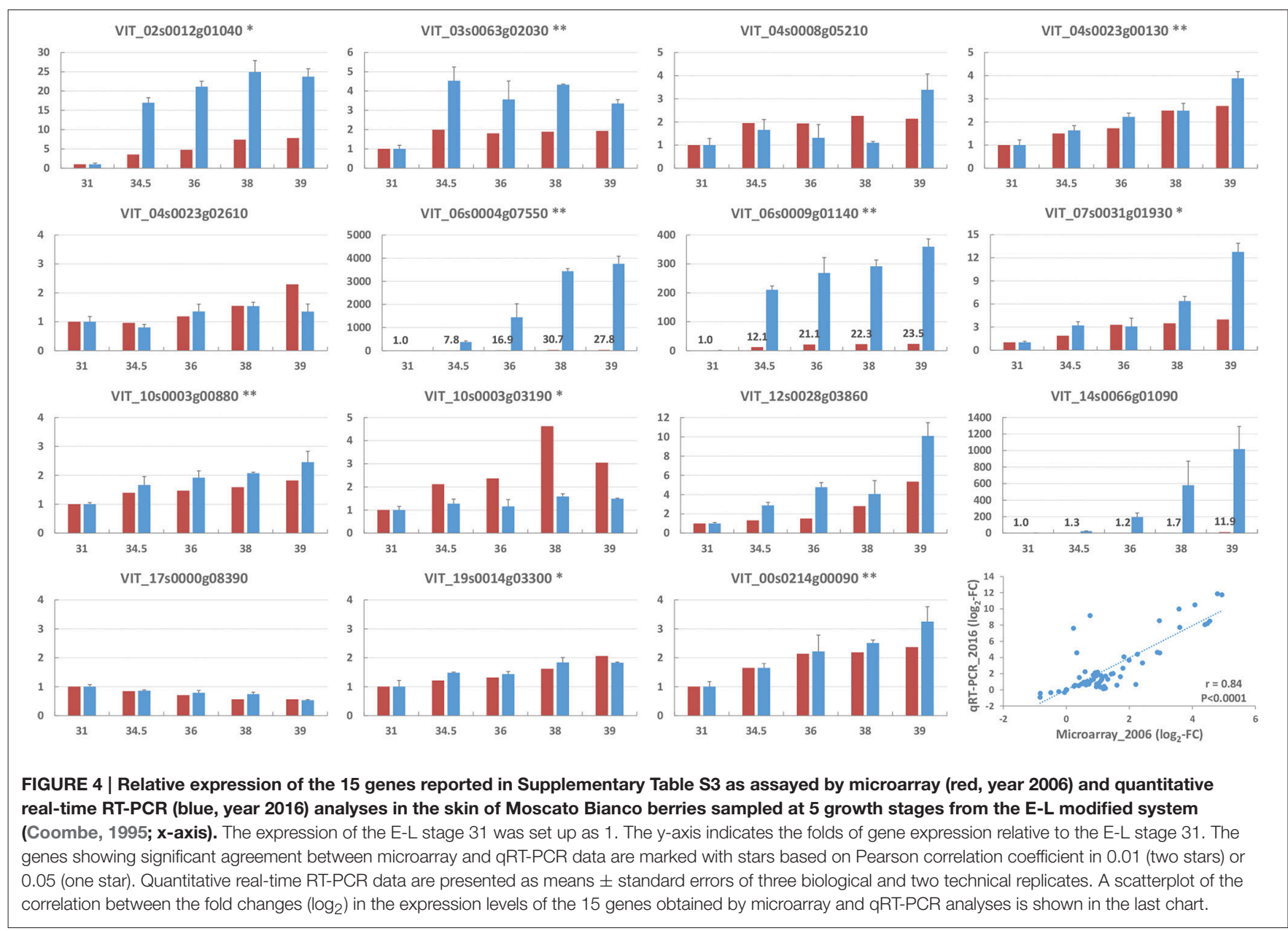

trend during berry ripening observed for $V v D X R$ in our study (VIT_17s0000g08390 in Figure 4) do not support a regulatory role, in agreement with Rodríguez-Concepción et al. (2001) and Mendoza-Poudereux et al. (2014). Oppositely, the expression of VvHDR (VIT_03s0063g02030 in Figure 4) is consistent with the veraison-initiated accumulation of monoterpenes, as reported by Martin et al. (2012) and Wen et al. (2015) (Table 2 and Supplementary Table S9).

Middle and late terpenoid pathway genes. In other plant species GPPS works as a heterodimeric complex; in particular, the levels of GPPS small subunit, but not GPPS large subunit, might play a key role in regulating monoterpene biosynthesis (Tholl et al., 2004). Consistently, the AROS probes for GPPS large subunit genes (VIT_04s0023g01210 and VIT_18s0001g12000) were neither differentially expressed during Moscato Bianco berry ripening nor correlated to any monoterpene. No probe could be identified for the GPPS small subunit.

Only three probes for terpene synthases are present on the Grape AROS V.1 array, which are not specific to any single gene prediction. One of them, showing the best match to the sesquiterpene synthases VIT_18s0001g04280 and VIT_18s0001g04530, was up-regulated during Moscato Bianco berry ripening (Table 2 and Supplementary Table S9). It is worth noting that the same genes were reported to correlate with linalool and $\alpha$-terpineol (Savoi et al., 2016).

An interesting candidate gene for the biosynthesis of monoterpenes is a nudix hydrolase (VIT_10s0003g00880), whose expression increases along berry development (Figure 4). The corresponding probe belongs to cluster 4, which also harbors several monoterpenes (Table 2, Supplementary Tables S8, S9). Recently, a rose nudix hydrolase was reported to convert geranyl diphosphate to geranyl monophosphate, which in turn is hydrolyzed to geraniol by a phosphatase activity (Magnard et al., 2015). This alternative and completely new terpene synthase-independent route for monoterpene production might play a role also in other plants, including grapevine.

\section{Secondary monoterpene transformations}

Extensive oxidative monoterpene metabolism has been reported in grapes and wine, with a percentage of linalool oxygenation ranging from 52 to $97 \%$ (Ilc et al., 2016b). The main linalool oxidation products are trans/cis-8-hydroxy-linalool (by hydroxylation), trans/cis pyranoid/furanoid linalool oxides and polyhydroxylated derivatives or polyols like the odorless hydroxy-diendiol I and II (by epoxidation and hydrolysis). Similarly, C-8 oxygenated geraniol and citronellol derivatives 


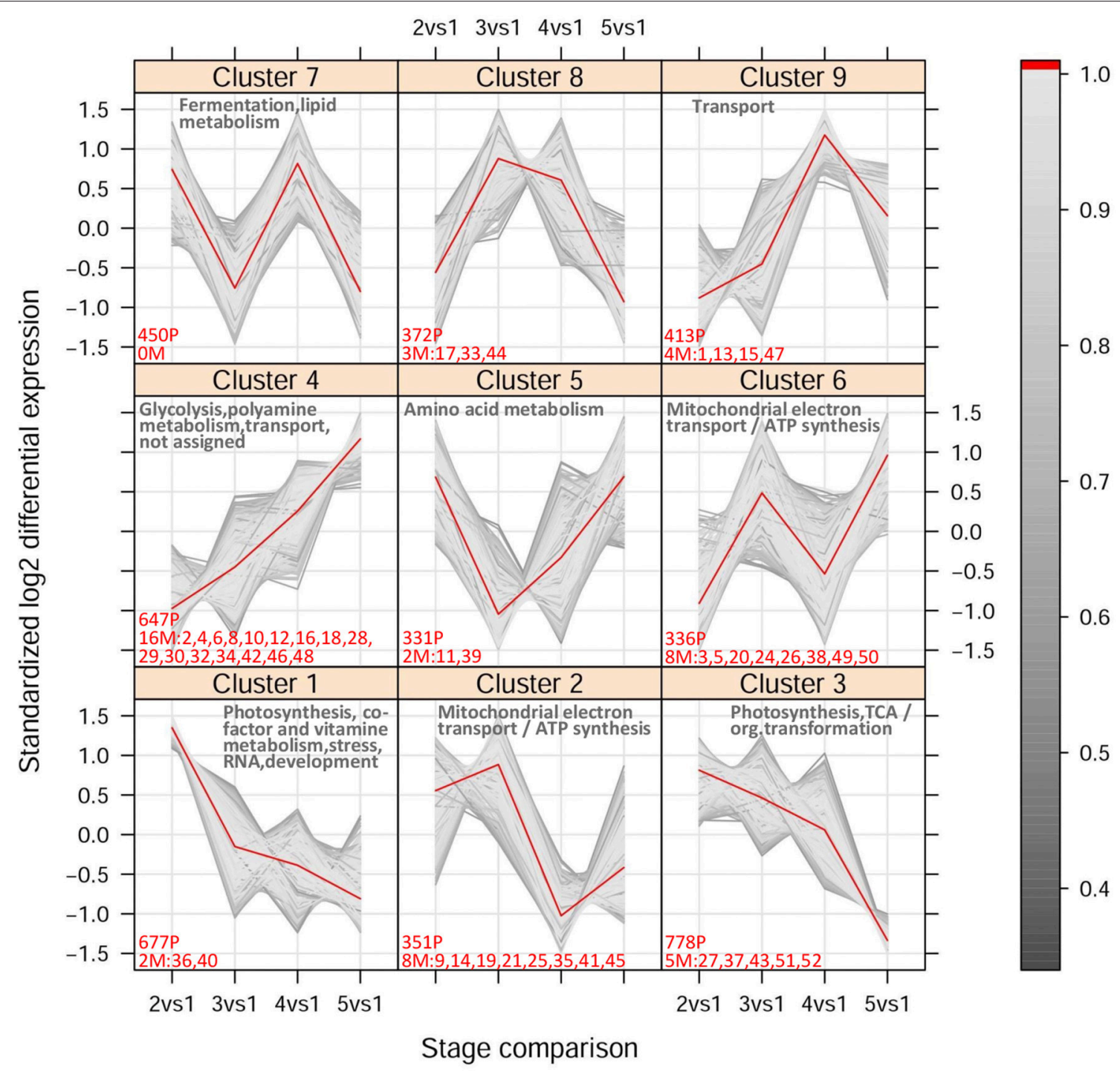

FIGURE 5 | Fuzzy c-means cluster output. The expression levels are represented as fold changes relative to stage 1 (pre-veraison). The time scale corresponds to the pairwise comparison between stages 2-5 and stage 1 in 2006. The color legend shows the cluster membership values calculated as described in Futschik and Carlisle (2005) and Kumar and Futschik (2007). The number of probes (P) and metabolites (M) included within each cluster (membership >0.5) is indicated in red. The names of metabolites are abbreviated as follows: 1 and $2=$ free and bound linalool, 3 and $4=$ free and bound geraniol, 5 and $6=$ free and bound nerol, 7 and $8=$ free and bound trans-8-hydroxy-linalool, 9 and $10=$ free and bound cis-8-hydroxy-linalool, 11 and $12=$ free and bound trans-furan linalool oxide (OxA), 13 and $14=$ free and bound cis-furan linalool oxide (OxB), 15 and $16=$ free and bound trans-pyran linalool oxide (OxC), 17 and $18=$ free and bound cis-pyran linalool oxide (OxD), 19 and 20 = free and bound trans-geranic acid, 21 and $22=$ free and bound citronellol, 23 and $24=$ free and bound $\alpha$-terpineol, 25 and $26=$ free and bound 4-terpineol, 27 and 28 = free and bound hydroxy-diendiol I + hydroxy-trienol, 29 and $30=$ free and bound hydroxy-diendiol II, 31 and $32=$ free and bound rose oxide I (cis isomer), 33 and $34=$ free and bound rose oxide II (trans isomer), 35 and $36=$ free and bound 3 -hydroxy- $\beta$-damascone, 37 and $38=$ free and bound 3 -oxo- $\alpha$-ionol, 39 and 40 = free and bound 6-methyl-5-hepten-2-one, 41 and $42=$ free and bound benzyl alcohol, 43 and $44=$ free and bound benzaldehyde, 45 and 46 = free and bound 2-phenylethanol, 47 and 48 = free and bound hexanol, 49 and 50 = free and bound trans-3-hexen-1-ol, 51 and $52=$ free and bound cis-3-hexen-1-ol. A summary of the Mapman functional categories over-represented within each cluster is also reported.

can be formed through hydroxylation, whereas the oxidation to geranial and neral (altogether named citral) is supposedly mediated by alcohol dehydrogenases. Geranic acid is another oxidation product of geraniol. Rose oxide is generated from citronellol by allylic hydroxylation and acid-catalyzed cyclization. Citronellol in turn arises from the reduction of geraniol and nerol (hydrogenation).
Members of the cytochrome P450 (CYP) 71 and 76 families were recently shown to metabolize linalool in Arabidopsis thaliana (Ginglinger et al., 2013; Höfer et al., 2014; Boachon et al., 2015). Interestingly, the CYP76 gene family has encountered an evident expansion in the grape genome (Nelson et al., 2008). In order to identify genes potentially implicated in grape monoterpenoid metabolism we looked 


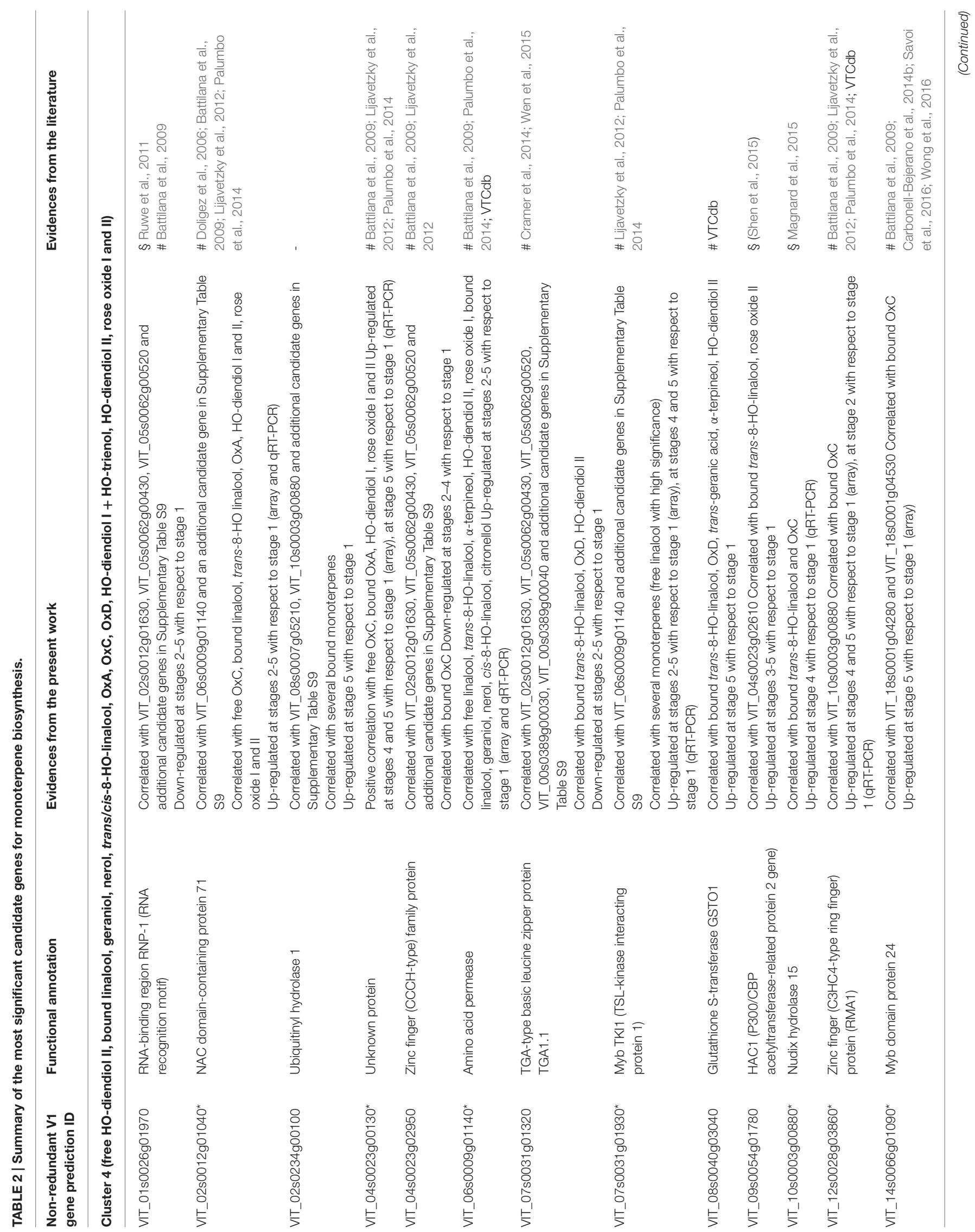




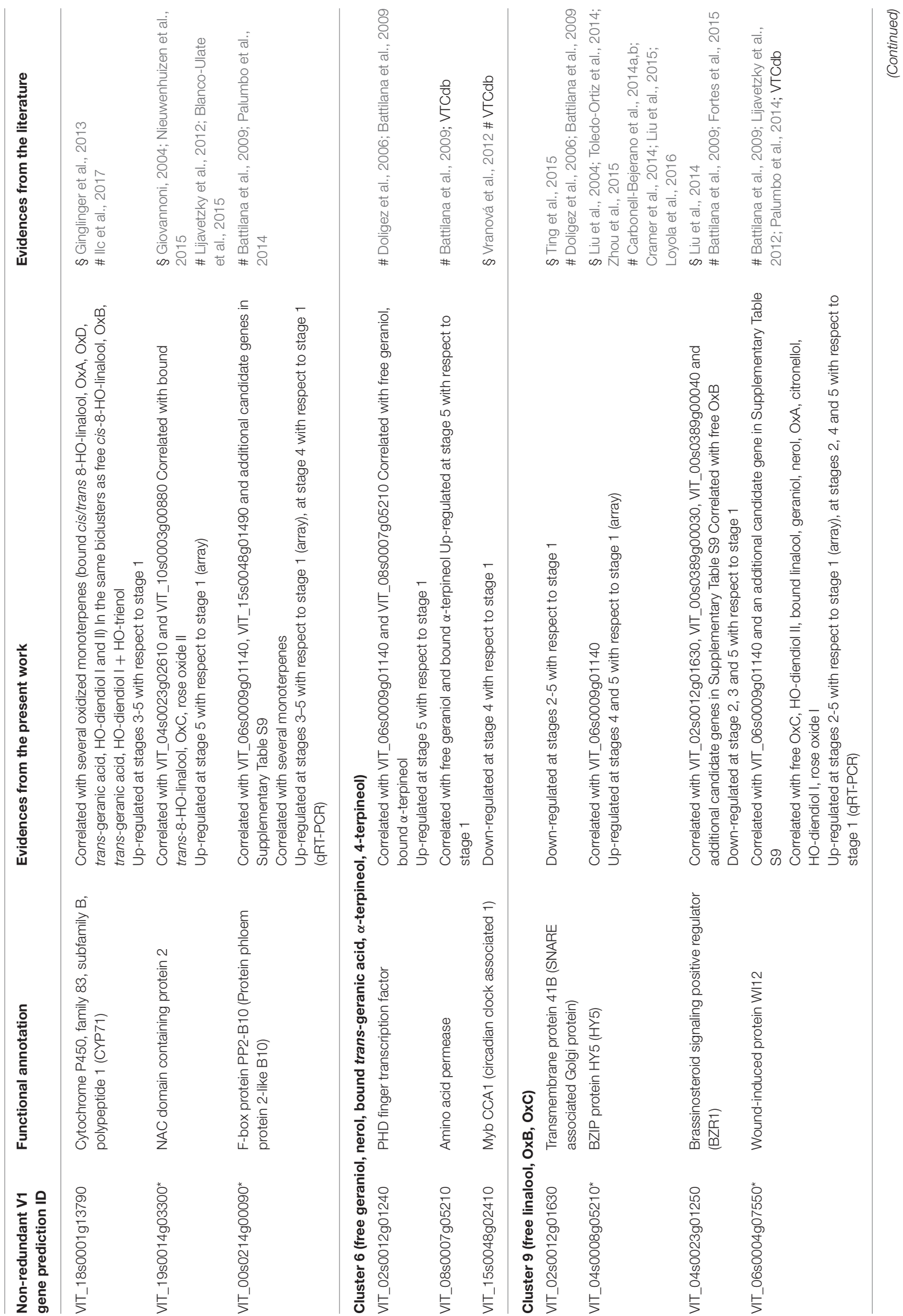




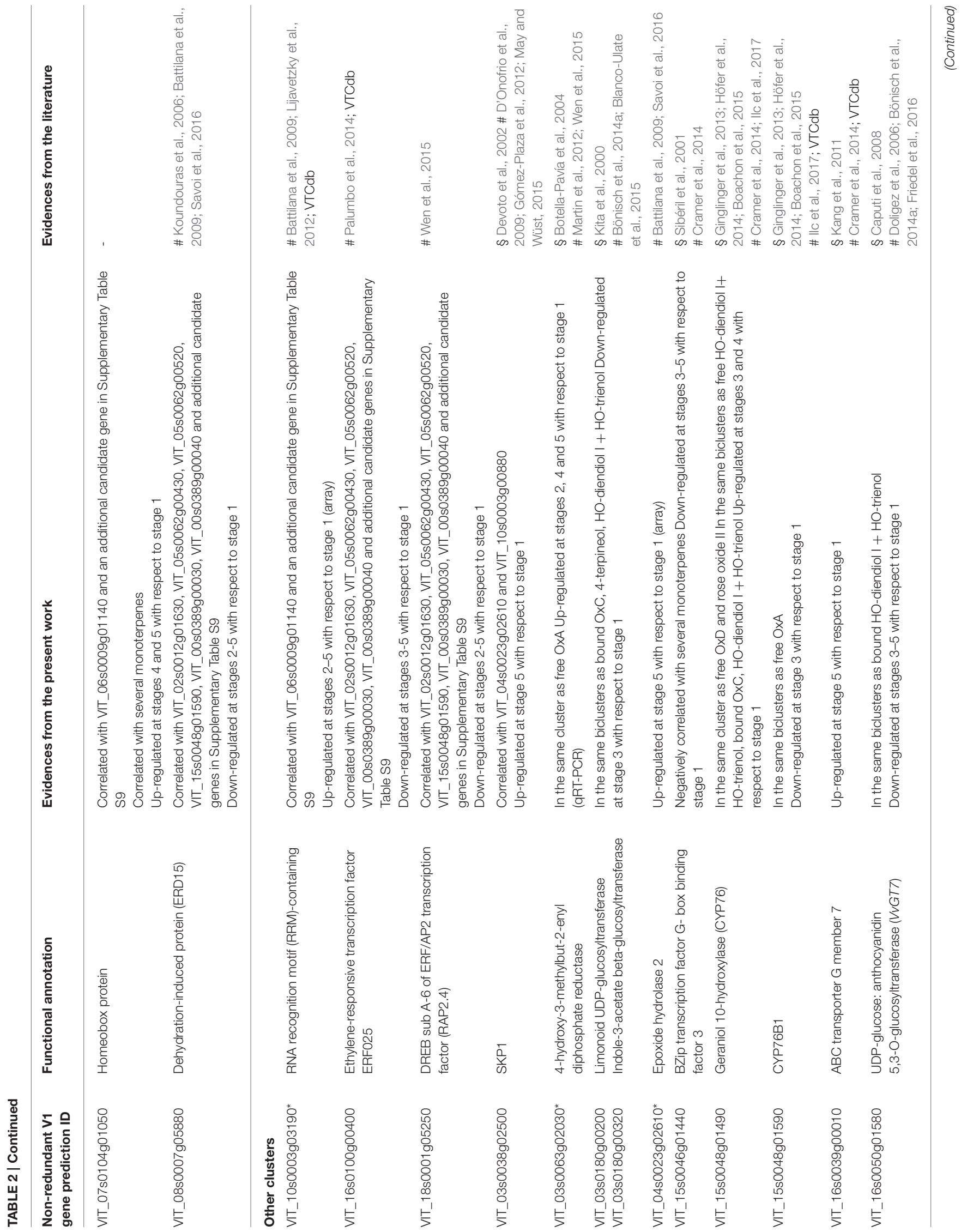




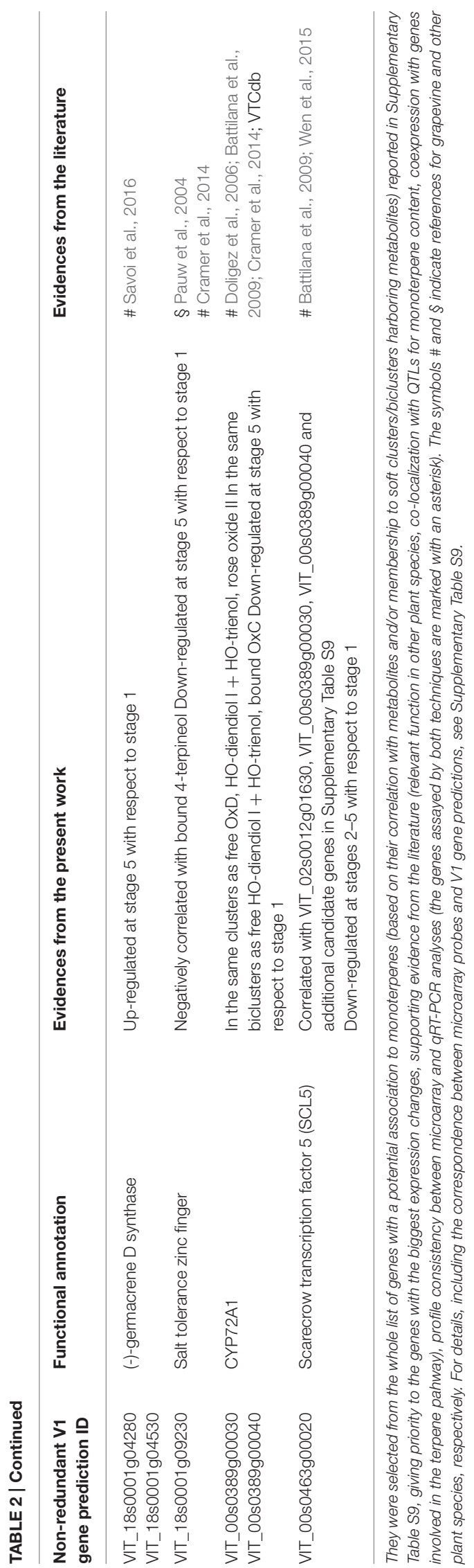

in VTCdb database (http://vtcdb.adelaide.edu.au/Home.aspx) for CYP genes coexpressed with linalool synthases, as in Ginglinger et al. (2013). This information was then added to our transcriptomic and metabolic integrated datasets. On this base, we propose some genes (VIT_15s0048g01490, VIT_15s0048g01590, VIT_18s0001g13790, VIT_00s0389g00030, VIT_00s0389g00040) and eventually additional ones as potential CYPs involved in linalool metabolism (Table 2 and Supplementary Table S9). Most of these candidates have been never reported elsewhere, and thus deserve further attention. Conversely, VIT_15s0048g01490 and VIT_18s0001g13790 were recently characterized by Ilc et al. (2017) but their biochemical activity was only tested on a limited number of compounds. Our findings suggest instead that these genes might play a role in the production of a broader set of hydroxylated and/or epoxidized products as in other species (Meesters et al., 2007; Ginglinger et al., 2013; Höfer et al., 2013, 2014; Boachon et al., 2015) and, even if the need for further oxidoreductases can not be excluded (Ilc et al., 2016a), they encourage to check this hypothesis by analyzing additional substrates (geraniol, nerol, citronellol) and products (e. g. pyranoid/furanoid linalool oxides, hydroxydiendiols, geranic acid, rose oxides) in CYP enzymatic assays. We also propose an epoxide hydrolase (VIT_04s0023g02610) to be assessed for involvement in monoterpene oxidative metabolism (Table 2 and Supplementary Table S9).

Based on their sequence similarity with terpenoid glucosyltransferases from different plant species and on their membership in biclusters harboring some glucosylated monoterpenes, we propose that VIT_03s0180g00200, VIT_03s0180g00320 (Table 2) and eventually other genes reported in Supplementary Table S9 (VIT_03s0091g00040, VIT_03s0180g00280, VIT_05s0062g00430, VIT_05s0062g00520, VIT_05s0062g00630, VIT_05s0062g00640) might code for enzymes that glucosylate monoterpenes along with additional metabolites. Most of these genes have been investigated in previous works but they were not considered as candidates for monoterpene glucosylation in view of their decreasing expression during berry development (Khater et al., 2011; Bönisch et al., 2014a,b). However, they might be involved in the production of glucosylated monoterpenes with a similar trend, like the high oxidation state monoterpenoids sharing the same biclusters (Table 2 and Supplementary Table S9), which were not quantified in those papers. This hypothesis is not contradicted by the lack of gene annotation referring to the "Monoterpenoid biosynthesis" pathway and of positive correlation between transcript and monoterpenyl glucoside accumulation, as the same holds for the biochemically characterized monoterpenol glucosyltransferase $V v G T 7$ (Table 2) and may be explained by the broad substrate tolerance and overlapping enzymatic activities of the large GT family. Monoterpenyl glucosides are only intermediates within the glycosylation pathway and post-transcriptional control is additionally involved (Bönisch et al., 2014a).

\section{Monoterpene transport}

Terpene transport within the cell and into the apoplast is an almost unexplored field. It may engage multiple pathways, e.g., (1) insertion of the hydrophobic terpenes into vesicle membranes 
followed by transport and fusion to the plasma membrane, (2) carrier proteins (like GSTs, glutathione S-transferases, and ABC, ATP-binding cassette transporters) that conduct these molecules to the (plasma) membrane, and (3) direct diffusion between the endoplasmic reticulum and/or plastidial (stromule) membranes and the plasma membrane (Ting et al., 2015). The fusion of vesicles with target membranes is mediated by a group of proteins called SNAREs (soluble NSF attachment protein receptors). Surprisingly and still without a clear underlying mechanism, both sesquiterpenes and monoterpenes were boosted when vesicle fusion was inhibited in Nicotiana benthamiana (Ting et al., 2015). Moreover, two Arabidopsis linalool synthases were detected in vesicular structures associated with the plastids (Ginglinger et al., 2013). Based on these findings, we included among our candidates a gene coding for a SNARE associated Golgi protein (VIT_02s0012g01630). Plant ABCG transporters play a role in the flux of secondary metabolites, particularly of terpenoid origin (Kang et al., 2011). Interestingly, we found an ABCG gene (VIT_16s0039g00010) that shows a profile consistent with monoterpene accumulation and is coexpressed with several monoterpene synthases in VTCdb. We also selected a glutathione S-transferase (VIT_08s0040g03040) and two amino acid permeases (VIT_06s0009g01140 and VIT_08s0007g05210), which are coexpressed with monoterpene biosynthetic genes in VTCdb and positively correlated to several monoterpenes in the present study (Table 2 and Supplementary Table S9).

\section{Monoterpene biosynthesis transcriptional regulation}

Recent works (Cramer et al., 2014; Wen et al., 2015) suggested that a group of ERF6-type transcription factors clustered on chromosome 16 are involved in aroma accumulation, based on the correlation of their transcript abundance and the transcript abundance of several terpenoid pathway genes. For some of these regulatory genes, e.g., the orthologs of CrORCA2, CrORCA3, and AaERF1 (De Geyter et al., 2012), no probe was found among the 4,450 probes used for our integrative analysis. Other ERF genes reported in the mentioned papers (VIT_16s0013g00950, VIT_16s0013g00980, VIT_16s0013g00990, VIT_16s0013g01030, VIT_16s0013g01050, VIT_16s0013g01060, not listed in Supplementary Table S9) belonged to clusters 1, 2, 7 and did not show any relevant positive correlation with monoterpenes. However, some of the AROS probes had only a partial match with these genes, as a consequence they might correspond instead to ERF gene isoforms not involved in flavor determination. Conversely, the genes VIT_16s0100g00400 and VIT_18s0001g05250 showed an expression profile consistent with the accumulation of monoterpenes in Moscato Bianco ripening berry (Table 2 and Supplementary Table S9).

We also observed an interesting behavior (Figure 4, Table 2, and Supplementary Table S9) for TFs of the MYB (VIT_14s0066g01090) and NAC (VIT_19s0014g03300) families that promote mono- and sequiterpene production in other plant species (Reeves et al., 2012; Nieuwenhuizen et al., 2015). In particular, VIT_14s0066g01090 (MYB24) has been proposed as a candidate transcriptional regulator of (mono)terpene biosynthesis also in grapevine (Matus, 2016; Savoi et al.,
2016), for which reason it deserves further attention. Finally, based on the negative effect of GBF1 (G-box binding factor 1) and ZCT (zinc-finger Catharanthus transcription factor) proteins on the expression of the TIA (terpenoid indole alkaloid) biosynthetic genes Str (strictosidine synthase) and Tdc (tryptophan decarboxylase) (Sibéril et al., 2001; Pauw et al., 2004), we selected two genes (VIT_15s0046g01440 and VIT_18s0001g09230) negatively correlated with monoterpene accumulation during Moscato Bianco berry ripening (Table 2 and Supplementary Table S9).

One of the signals dramatically impacting isoprenoid biosynthesis in higher plants is light, which activates the MEP pathway at the transcriptional and post-transcriptional level (Rodríguez-Concepción, 2006; Cordoba et al., 2009; Vranová et al., 2012; Mannen et al., 2014). Sunlight exclusion limits the synthesis and accumulation of terpenes also in grape berries (linalool and the bound forms being the most responsive) by especially affecting DXS and TPS genes (Zhang et al., 2014; Friedel et al., 2016; Joubert et al., 2016; Matus, 2016; Sasaki et al., 2016). Our findings (Figure 4, Table 2, and Supplementary Table S9) are consistent with a role, among others, for HY5 (LONG HYPOCOTYL5, VIT_04s0008g05210) in the regulation of light-induced terpenoid biosynthesis in grapes, in agreement with other evidences (CarbonellBejerano et al., 2014a,b; Zhou et al., 2015; Loyola et al., 2016).

The isoprenoid pathway has also been reported to be under the circadian clock control. In particular, the emission of volatile terpenoids follows a diurnal rhythm and genes encoding enzymes involved in IPP biosynthesis (especially those from the MEP pathway) and downstream pathways are coexpressed with circadian clock genes and show typical circadian expression profiles (Cordoba et al., 2009; Vranová et al., 2012; Pokhilko et al., 2015). Some probes on the AROS array correspond to a gene of the circadian oscillator (VIT_15s0048g02410) and fall into clusters harboring several monoterpenes (Table 2 and Supplementary Table S9).

The expression profile of a number of additional transcription factors (including master regulators) and genes potentially involved in the post-transcriptional regulation (Hemmerlin, 2013) overlaps monoterpene accumulation during Moscato Bianco berry ripening, which supports a role in the control of monoterpene biosynthesis for VIT_01s0026g01970, VIT_02s0012g01040, VIT_02s0012g01240, VIT_02s0234g00100, VIT_03s0038g02500, VIT_04s0023g00130, VIT_04s0023g01250, VIT_04s0023g02950, VIT_06s0004g07550, VIT_07s0031g01320, VIT_07s0031g01930, VIT_07s0104g01050, VIT_08s0007g05880, VIT_09s0054g01780, VIT_10s0003g03190, VIT_12s0028g03860, VIT_00s0214g00090, VIT_00s0463g00020 (Figure 4, Table 2, Supplementary Table S9). To our knowledge, these genes represent new regulatory candidates for the production of several (cluster 4) or specific metabolites, like linalool (cluster 9) and geraniol/nerol (cluster 6), as suggested by their co-localization with QTLs and their correlation with enzyme/transporter genes correlated to metabolites. 


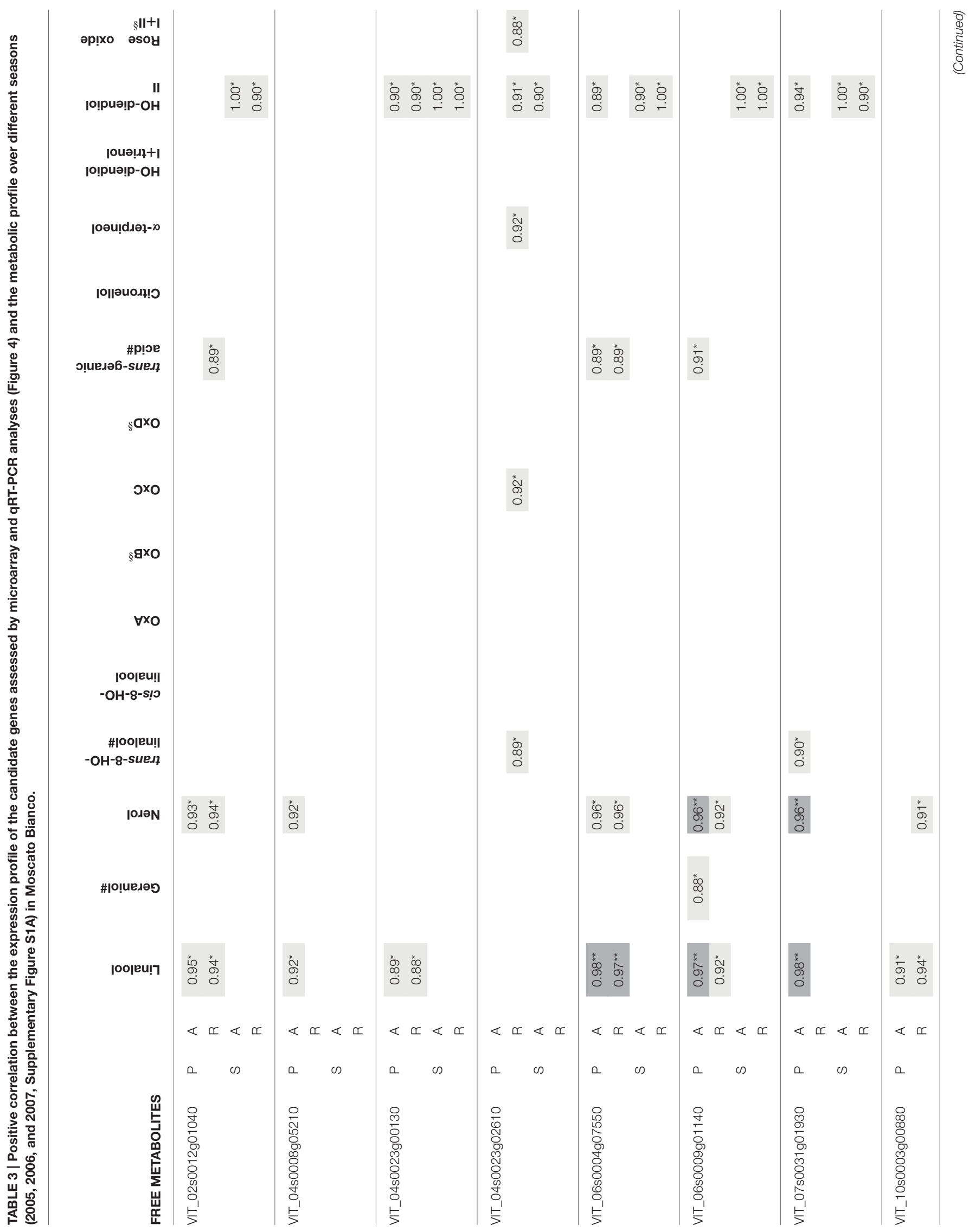




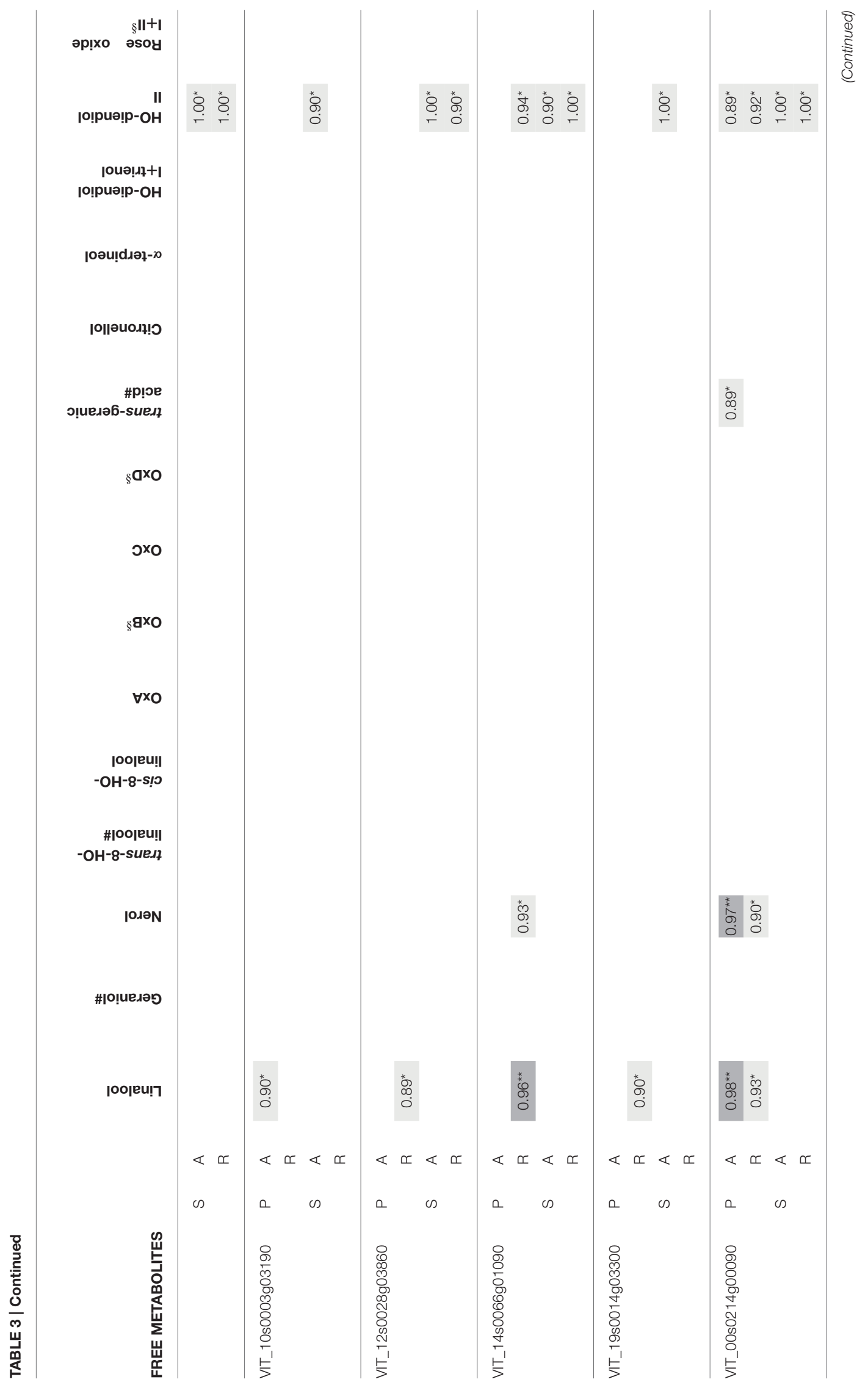




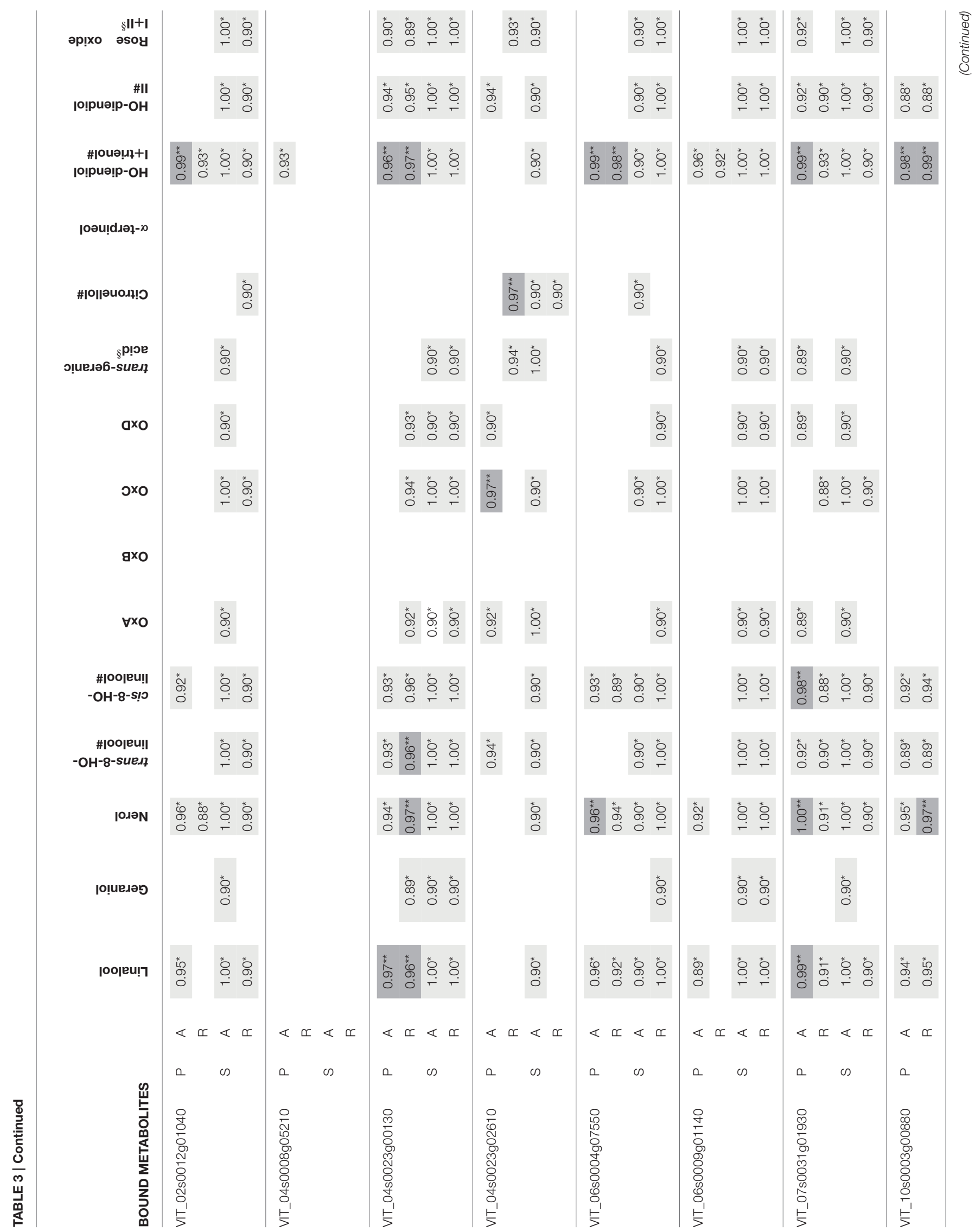




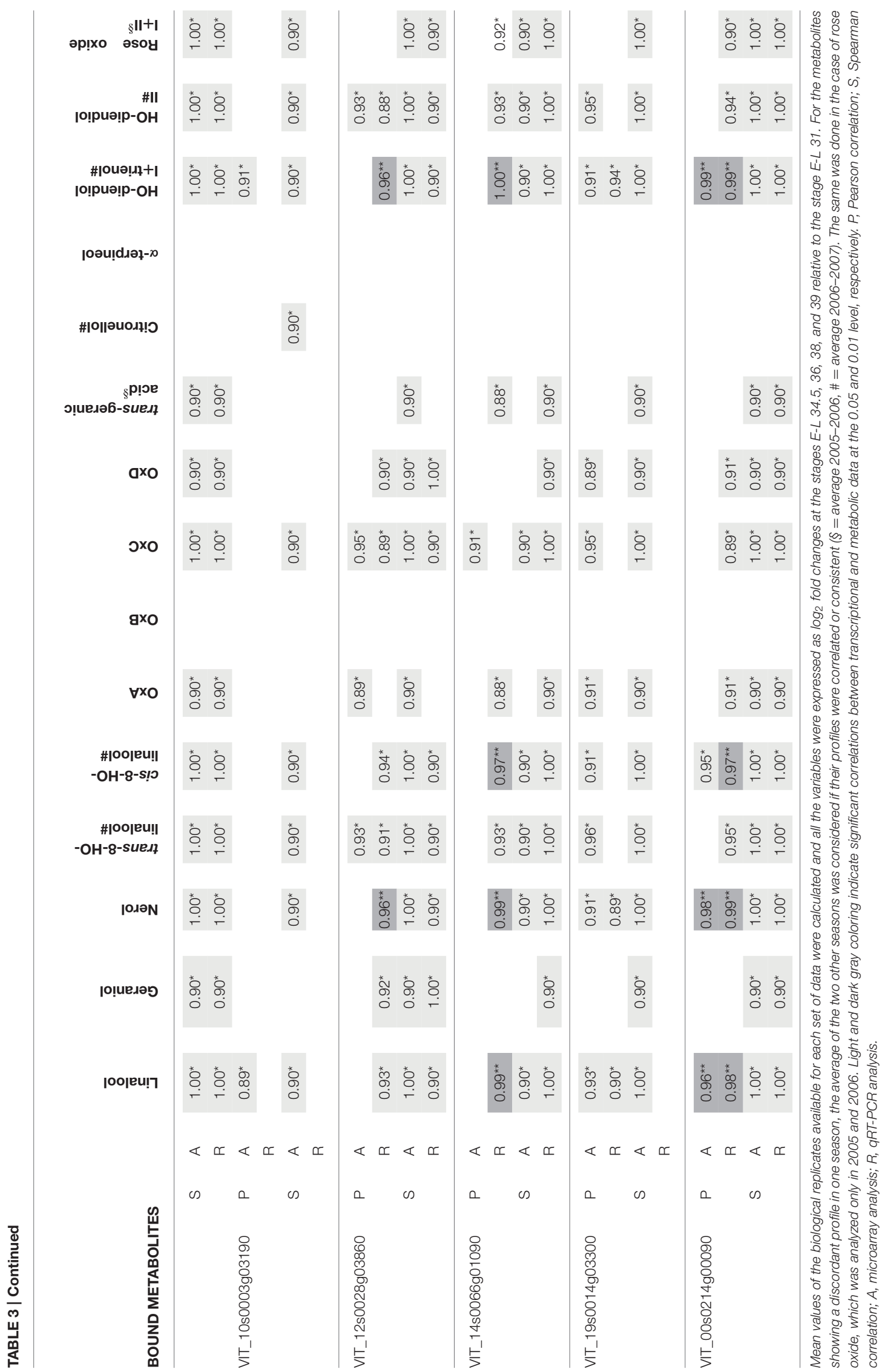




\section{Integration of Transcriptomic and Metabolic Data Over Multiple Seasons to Verify a Subset of Candidate Genes}

In order to confirm the above links between transcriptome and metabolome in aroma development, the 15 genes assessed by both microarray and real-time analyses were also tested for correlation with the metabolic profile over three seasons, which were considered as three biological replicates (Table 3 and Supplementary Figure S1A). Significant correlations were found for all the genes except $V v D X R$ (confirming the results from 2006 data) and $V v H D R$, which probably precedes monoterpene accumulation. Several compounds were affected, especially in their glycosidically bound form. Unsurprisingly, most of the metabolites with no correlation showed an inconsistent profile among seasons (e.g., free OxA and citronellol, bound $\alpha$-terpineol) or a decreasing trend along berry ripening (e.g., free HOdiendiol I + HO-trienol and bound $\mathrm{OxB}$ ). Though not ensuring a punctual conformity to the observations from a single year (Table 2), the findings from multiple seasons (Table 3) prove the general consistency of the outcomes of different techniques and years and argue for the reliability of the whole set of results based on the integration of 2006 transcriptomic and metabolic data.

\section{CONCLUSION}

Understanding the origin of grape aromatic compounds is essential in the breeding of new varieties and in the management of high-quality crops in a changing climate. In this work, previously undescribed gene-to-metabolite networks with a possible association to grape flavor were deduced by integrating the expression profiles of 4,450 gene tags and the accumulation profiles of 52 metabolites. Pairwise correlation and clustering methods pointed to several structural and regulatory genes potentially involved in the biosynthesis of monoterpenes, which

\section{REFERENCES}

Agudelo-Romero, P., Erban, A., Sousa, L., Pais, M. S., Kopka, J., and Fortes, A. M. (2013). Search for transcriptional and metabolic markers of grape pre-ripening and ripening and insights into specific aroma development in three portuguese cultivars. PLoS ONE 8:e60422. doi: 10.1371/journal.pone.0060422

Battilana, J. (2009). Genetic Determination of Aroma in Grapevine (Vitis vinifera L.). From QTL to Gene Expression Analysis in Aromatic and Non-aromatic Varieties. [dissertation/Ph.D. thesis], University of Padova, Padova.

Battilana, J., Costantini, L., Emanuelli, F., Sevini, F., Segala, C., Moser, S., et al. (2009). The 1-deoxy-D-xylulose 5-phosphate synthase gene co-localizes with a major QTL affecting monoterpene content in grapevine. Theor. Appl. Genet. 118, 653-669. doi: 10.1007/s00122-008-0927-8

Battilana, J., Emanuelli, F., Gambino, G., Gribaudo, I., Gasperi, F., Boss, P. K., et al. (2011). Functional effect of grapevine 1-deoxy-D-xylulose 5-phosphate synthase substitution $\mathrm{K} 284 \mathrm{~N}$ on Muscat flavour formation. J. Exp. Bot. 62, 5497-5508. doi: 10.1093/jxb/err231

Benjamini, Y., and Hochberg, Y. (1995). Controlling the false discovery rate: a practical and powerful approach to multiple testing. J. R. Statist. Soc. B 57, 289-300.

Black, C. A., Parker, M., Siebert, T. E., Capone, D. L., and Francis, I. L. (2015). Terpenoids and their role in wine flavour: recent advances. Aust. J. Grape Wine R. 21, 582-600. doi: 10.1111/ajgw.12186 paves the way for locating candidates for at least some of the missing links in the underlying pathway. Our collective findings contribute toward understanding the regulation of secondary metabolism in Muscat-type grape cultivars through the formulation of testable hypotheses regarding the function of specific genes.

\section{AUTHOR CONTRIBUTIONS}

LC, CK, JB, FE, SD, and MG contributed to the project design; LC, MT, JB, FE, MS, and CC took part in the experimental work; RL provided the metabolic analysis; LC, CK, and MM performed the statistical and bioinformatic analyses; LC and CK were involved in data interpretation; LC wrote the manuscript. All the authors approved the final version of this text.

\section{FUNDING}

This research was sustained by a Short Term Scientific Mission grant awarded to LC by the Institute of Vine and Wine Sciences (Bordeaux, France) and with the financial support provided by the Autonomous Province of Trento (Accordo di Programma).

\section{ACKNOWLEDGMENTS}

We thank Silvia Lorenzi for sample collection, Sergio Moser for metabolic analysis and Pietro Franceschi for statistical support.

\section{SUPPLEMENTARY MATERIAL}

The Supplementary Material for this article can be found online at: http://journal.frontiersin.org/article/10.3389/fpls.2017. 00780/full\#supplementary-material

Blanco-Ulate, B., Amrine, K. C. H., Collins, T. S., Rivero, R. M., Vicente, A. R., Morales-Cruz, A., et al. (2015). Developmental and metabolic plasticity of white-skinned grape berries in response to Botrytis cinerea during noble rot. Plant Physiol. 169, 2422-2443. doi: 10.1104/pp.15.00852

Boachon, B., Junker, R. R., Miesch, L., Bassard, J. E., Höfer, R., Caillieaudeaux, R., et al. (2015). CYP76C1 (Cytochrome P450)-mediated linalool metabolism and the formation of volatile and soluble linalool oxides in Arabidopsis flowers: a strategy for defense against floral antagonists. Plant Cell 27, 2972-2990. doi: $10.1105 /$ tpc. 15.00399

Bönisch, F., Frotscher, J., Stanitzek, S., Rühl, E., Wüst, M., Bitz, O., et al. (2014a). A UDP-glucose:monoterpenol glucosyltransferase adds to the chemical diversity of the grapevine metabolome. Plant Physiol. 165, 561-581. doi: 10.1104/pp.113.232470

Bönisch, F., Frotscher, J., Stanitzek, S., Rühl, E., Wüst, M., Bitz, O., et al. (2014b). Activity-based profiling of a physiologic aglycone library reveals sugar acceptor promiscuity of family 1 UDP-glucosyltransferases from grape. Plant Physiol. 166, 23-39. doi: 10.1104/pp.114.242578

Botella-Pavía, P., Besumbes, O., Phillips, M. A., Carretero-Paulet, L., Boronat, A., and Rodríguez-Concepción, M. (2004). Regulation of carotenoid biosynthesis in plants: evidence for a key role of hydroxymethylbutenyl diphosphate reductase in controlling the supply of plastidial isoprenoid precursors. Plant J. 40, 188-199. doi: 10.1111/j.1365-313X.2004. 02198.x 
Caputi, L., Lim, E. K., and Bowles, D. J. (2008). Discovery of new biocatalysts for the glycosylation of terpenoid scaffolds. Chem. Eur. J. 14, 6656-6662. doi: $10.1002 /$ chem. 200800548

Carbonell-Bejerano, P., Diago, M. P., Martínez-Abaigar, J., Martínez-Zapater, J. M., Tardáguila, J., and Núñez-Olivera, E. (2014b). Solar ultraviolet radiation is necessary to enhance grapevine fruit ripening transcriptional and phenolic responses. BMC Plant Biol. 14:183. doi: 10.1186/1471-2229-14-183

Carbonell-Bejerano, P., Rodríguez, V., Royo, C., Hernáiz, S., MoroGonzález, L. C., Torres-Viñals, M., et al. (2014a). Circadian oscillatory transcriptional programs in grapevine ripening fruits. BMC Plant Biol. 14:78. doi: 10.1186/1471-2229-14-78

Churchill, G. A. (2002). Fundamentals of experimental design for cDNA microarrays. Nat. Genet. 32, 490-495. doi: 10.1038/ng1031

Coombe, B. G. (1995). Growth stages of the grapevine: adoption of a system for identifying grapevine growth stages. Aust. J. Grape Wine R. 1, 100-110. doi: 10.1111/j.1755-0238.1995.tb00086.x

Cordoba, E., Salmi, M., and León, P. (2009). Unravelling the regulatory mechanisms that modulate the MEP pathway in higher plants. J. Exp. Bot. 60, 2933-2943. doi: 10.1093/jxb/erp190

Costantini, L., Malacarne, G., Lorenzi, S., Troggio, M., Mattivi, F., Moser, C., et al. (2015). New candidate genes for the fine regulation of the colour of grapes. J. Exp. Bot. 66, 4427-4440. doi: 10.1093/jxb/erv159

Cramer, G. R., Ghan, R., Schlauch, K. A., Tillett, R. L., Heymann, H., Ferrarini, A., et al. (2014). Transcriptomic analysis of the late stages of grapevine (Vitis vinifera cv. Cabernet Sauvignon) berry ripening reveals significant induction of ethylene signaling and flavor pathways in the skin. BMC Plant Biol. 14:370. doi: 10.1186/s12870-014-0370-8

D'Onofrio, C., Cox, A., Davies, C., and Boss, P. K. (2009). Induction of secondary metabolism in grape cell cultures by jasmonates. Funct. Plant Biol. 36, 1-16. doi: 10.1071/FP08280

De Geyter, N., Gholami, A., Goormachtig, S., and Goossens, A. (2012). Transcriptional machineries in jasmonate-elicited plant secondary metabolism. Trends Plant Sci. 17, 349-359. doi: 10.1016/j.tplants.2012.03.001

Devoto, A., Nieto-Rostro, M., Xie, D., Ellis, C., Harmston, R., Patrick, E., et al. (2002). COI1 links jasmonate signalling and fertility to the SCF ubiquitin-ligase complex in Arabidopsis. Plant J. 32, 457-466. doi: 10.1046/j.1365-313X.2002.01432.x

Doligez, A., Audiot, E., Baumes, R., and This, P. (2006). QTLs for muscat flavor and monoterpenic odorant content in grapevine (Vitis vinifera L.). Mol. Breed. 18, 109-125. doi: 10.1007/s11032-006-9016-3

D'Onofrio, C., Matarese, F., and Cuzzola, A. (2016). Study of the terpene profile at harvest and during berry development of Vitis vinifera L. aromatic varieties Aleatico, Brachetto, Malvasia di Candia aromatica and Moscato bianco. J. Sci. Food Agric. doi: 10.1002/jsfa.8126. [Epub ahead of print].

Duchêne, E., Butterlin, G., Claudel, P., Dumas, V., Jaegli, N., and Merdinoglu, D. (2009). A grapevine (Vitis vinifera L.) deoxy-d-xylulose synthase gene colocates with a major quantitative trait loci for terpenol content. Theor. Appl. Genet. 118, 541-552. doi: 10.1007/s00122-008-0919-8

Dudareva, N., Klempien, A., Muhlemann, J. K., and Kaplan, I. (2013). Biosynthesis, function and metabolic engineering of plant volatile organic compounds. New Phytol. 198, 16-32. doi: 10.1111/nph.12145

Dunlevy, J. D., Kalua, C. M., Keyzers, R. A., and Boss, P. K. (2009). “The production of flavour and aroma compounds in grape berries," in Grapevine Molecular Physiology and Biotechnology, ed K. A. Roubelakis-Angelakis (Dordrecht: Springer), 293-340.

Ebang-Oke, J. P., de Billerbeck, G. M., and Ambid, C. (2003). “Temporal expression of the Lis gene from Vitis vinifera L., cv. Muscat de Frontignan," in Flavour Research at the Dawn of the Twenty-first Century, Proceedings of the 10th Weurman Flavour Research Symposium, June 25-28, 2002, eds J. L. Le Quere and P. X. Etievant (Beaune: Lavoisier), 321-325.

Ebeler, S. E., and Thorngate, J. H. (2009). Wine chemistry and flavor: looking into the crystal glass. J. Agric. Food Chem. 57, 8098-8108. doi: 10.1021/jf9000555

Emanuelli, F., Battilana, J., Costantini, L., Le Cunff, L., This, P., and Grando, M. S. (2010). A candidate gene association study for muscat flavor in grapevine Vitis vinifera L. BMC Plant Biol. 10:241. doi: 10.1186/1471-2229-10-241

Fortes, A. M., Agudelo-Romero, P., Silva, M. S., Ali, K., Sousa, L., Maltese, F., et al. (2011). Transcript and metabolite analysis in Trincadeira cultivar reveals novel information regarding the dynamics of grape ripening. BMC Plant Biol. 11:149. doi: 10.1186/1471-2229-11-149

Fortes, A. M., Teixeira, R. T., and Agudelo-Romero, P. (2015). Complex interplay of hormonal signals during grape berry ripening. Molecules 20, 9326-9343. doi: 10.3390/molecules20059326

Friedel, M., Frotscher, J., Nitsch, M., Hofmann, N., Bogs, J., Stoll, M., et al. (2016). Light promotes expression of monoterpene and flavonol metabolic genes and enhances flavour of winegrape berries (Vitis vinifera L. cv. Riesling). Aust. J. Grape Wine Res. 22, 409-421. doi: 10.1111/ajgw.12229

Futschik, M. E., and Carlisle, B. (2005). Noise-robust soft clustering of gene expression time-course data. J. Bioinform. Comput. Biol. 3, 965-988. doi: 10.1142/S0219720005001375

Ginglinger, J. F., Boachon, B., Höfer, R., Paetz, C., Köllner, T. G., Miesch, L. et al. (2013). Gene coexpression analysis reveals complex metabolism of the monoterpene alcohol linalool in Arabidopsis flowers. Plant Cell 25, 4640-4657. doi: $10.1105 /$ tpc.113.117382

Giovannoni, J. J. (2004). Genetic regulation of fruit development and ripening. Plant Cell 16, 170-180. doi: 10.1105/tpc.019158

Gómez-Plaza, E., Mestre-Ortuño, L., Ruiz-García, Y., Fernández-Fernández, J. I., and López-Roca, J. M. (2012). Effect of benzothiadiazole and methyl jasmonate on the volatile compound composition of Vitis vinifera L. Monastrell grapes and wines. Am. J. Enol. Vitic. 63, 394-401. doi: 10.5344/ajev.2012.12011

Grimplet, J., Van Hemert, J., Carbonell-Bejerano, P., Díaz-Riquelme, J., Dickerson, J., Fennell, A., et al. (2012). Comparative analysis of grapevine whole-genome gene predictions, functional annotation, categorization and integration of the predicted gene sequences. BMC Res. Notes 5:213. doi: 10.1186/1756-0500-5-213

Günata, Y. Z., Bayonove, C. L., Baumes, R. L., and Cordonnier, R. A. (1985). The aroma of grapes. II. Localisation and evolution of free and bound fractions of some grape aroma components c.v. Muscat during first development and maturation. J. Sci. Food Agr. 36, 857-862. doi: 10.1002/jsfa.2740360915

Guth, H. (1997). Identification of character impact odorants of different white wine varieties. J. Agric. Food Chem. 45, 3022-3026. doi: 10.1021/jf9608433

Hemmerlin, A. (2013). Post-translational events and modifications regulating plant enzymes involved in isoprenoid precursor biosynthesis. Plant Sci. 203204, 41-54. doi: 10.1016/j.plantsci.2012.12.008

Hjelmeland, A. K., and Ebeler, S. E. (2015). Glycosidically bound volatile aroma compounds in grapes and wine: a review. Am. J. Enol. Vitic. 66, 1-11. doi: 10.5344/ajev.2014.14104

Höfer, R., Boachon, B., Renault, H., Gavira, C., Miesch, L., Iglesias, J., et al. (2014). Dual function of the cytochrome P450 CYP76 family from Arabidopsis thaliana in the metabolism of monoterpenols and phenylurea herbicides. Plant Physiol. 166, 1149-1161. doi: 10.1104/pp.114.244814

Höfer, R., Dong, L., André, F., Ginglinger, J. F., Lugan, R., Gavira, C., et al. (2013). Geraniol hydroxylase and hydroxygeraniol oxidase activities of the CYP76 family of cytochrome P450 enzymes and potential for engineering the early steps of the (seco)iridoid pathway. Metab. Eng. 20, 221-232. doi: 10.1016/j.ymben.2013.08.001

Ilc, T., Halter, D., Miesch, L., Lauvoisard, F., Kriegshauser, L., Ilg, A., et al. (2017). A grapevine cytochrome $\mathrm{P} 450$ generates the precursor of wine lactone, a key odorant in wine. New Phytol. 213, 264-274. doi: 10.1111/nph.14139

Ilc, T., Parage, C., Boachon, B., Navrot, N., and Werck-Reichhart, D. (2016a). Monoterpenol oxidative metabolism: role in plant adaptation and potential applications. Front. Plant Sci. 7:509. doi: 10.3389/fpls.2016.00509

Ilc, T., Werck-Reichhart, D., and Navrot, N. (2016b). Meta-analysis of the core aroma components of grape and wine aroma. Front. Plant Sci. 7:1472. doi: 10.3389/fpls.2016.01472

Joubert, C., Young, P. R., Eyéghé-Bickong, H. A., and Vivier, M. A. (2016). Field-grown grapevine berries use carotenoids and the associated xanthophyll cycles to acclimate to UV exposure differentially in high and low light (shade) conditions. Front. Plant Sci. 7:786. doi: 10.3389/fpls.2016.00786

Kang, J., Park, J., Choi, H., Burla, B., Kretzschmar, T., Lee, Y., et al. (2011). Plant ABC transporters. Arabidopsis Book 9:e0153. doi: 10.1199/tab.0153

Khater, F., Fournand, D., Vialet, S., Meudec, E., Cheynier, V., and Terrier, N. (2011). Identification and functional characterization of cDNAs coding for hydroxybenzoate/hydroxycinnamate glucosyltransferases co-expressed with genes related to proanthocyanidin biosynthesis. J Exp. Bot. 63, 1201-1214. doi: $10.1093 /$ jxb/err340 
Kita, M., Hirata, Y., Moriguchi, T., Endo-Inagaki, T., Matsumoto, R., Hasegawad, S., et al. (2000). Molecular cloning and characterization of a novel gene encoding limonoid UDP-glucosyltransferase in Citrus. Febs Lett. 469, 173-178. doi: 10.1016/S0014-5793(00)01275-8

Koundouras, S., Marinos, V., Gkoulioti, A., Kotserisis, Y., and Van Leeuwen, C. (2006). Influence of vineyard location and vine water status on fruit maturation of non-irrigated cv. Agiorgitiko (Vitis vinifera L.). Effects on wine phenolic and aroma components. J. Agric. Food Chem. 54, 5077-5086. doi: 10.1021/jf0605446

Kumar, L., and Futschik, M. (2007). Mfuzz: a software package for soft clustering of microarray data. Bioinformation 2, 5-7. doi: 10.6026/97320630002005

Lemoine, S., Combes, F., Servant, N., and Le Crom, S. (2006). Goulphar: rapid access and expertise for standard two-color microarray normalization methods. BMC Bioinform. 7:467. doi: 10.1186/1471-2105-7-467

Li, G., Ma, Q., Tang, H., Paterson, A. H., and Xu, Y. (2009). QUBIC: a qualitative biclustering algorithm for analyses of gene expression data. Nucl. Acids Res. 37:e101. doi: 10.1093/nar/gkp491

Liberman, L. M., Sozzani, R., and Benfey, P. N. (2012). Integrative systems biology: an attempt to describe a simple weed. Curr. Opin. Plant Biol. 15, 162-167. doi: 10.1016/j.pbi.2012.01.004

Lijavetzky, D., Carbonell-Bejerano, P., Grimplet, J., Bravo, G., Flores, P., Fenoll, J., et al. (2012). Berry flesh and skin ripening features in Vitis vinifera as assessed by transcriptional profiling. PLoS ONE 7:e39547. doi: 10.1371/journal.pone.0039547

Liu, L., Gregan, S., Winefield, C., and Jordan, B. (2015). From UVR8 to flavonol synthase: UV-B-induced gene expression in Sauvignon blanc grape berry. Plant Cell Environ. 38, 905-919. doi: 10.1111/pce.12349

Liu, L., Jia, C., Zhang, M., Chen, D., Chen, S., Guo, R., et al. (2014). Ectopic expression of a BZR1-1D transcription factor in brassinosteroid signalling enhances carotenoid accumulation and fruit quality attributes in tomato. Plant Biotech. J. 12, 105-115. doi: 10.1111/pbi.12121

Liu, Y., Roof, S., Ye, Z., Barry, C., van Tuinen, A., Vrebalov, J., et al. (2004). Manipulation of light signal transduction as a means of modifying fruit nutritional quality in tomato. Proc. Natl. Acad. Sci. U.S.A. 101, 9897-9902. doi: 10.1073/pnas.0400935101

Loyola, R., Herrera, D., Mas, A., Wong, D. C., Höll, J., Cavallini, E., et al. (2016). The photomorphogenic factors UV-B RECEPTOR 1, ELONGATED HYPOCOTYL 5, and HY5 HOMOLOGUE are part of the UV-B signalling pathway in grapevine and mediate flavonol accumulation in response to the environment. J. Exp. Bot. 67, 5429-5445. doi: 10.1093/jxb/erw307

Luan, F., Hampel, D., Mosandl, A., and Wüst, M. (2004). Enantioselective analysis of free and glycosidically bound monoterpene polyols in Vitis vinifera L. cvs. Morio Muscat and Muscat Ottonel: evidence for an oxidative monoterpene metabolism in grapes. J. Agric. Food Chem. 52, 2036-2041. doi: 10.1021/jf030701q

Luan, F., Mosandl, A., Degenhardt, A., Gubesch, M., and Wüst, M. (2006a). Metabolism of linalool and substrate analogs in grape berry mesocarp of Vitis vinifera L. cv. Morio Muscat: demonstration of stereoselective oxygenation and glycosylation. Anal. Chim. Acta 563, 353-364. doi: 10.1016/j.aca.2006.01.001

Luan, F., Mosandl, A., Gubesch, M., and Wüst, M. (2006b). Enantioselective analysis of monoterpenes in different grape varieties during berry ripening using stir bar sorptive extraction- and solid phase extraction-enantioselectivemultidimensional gas chromatography-mass spectrometry. J. Chromatogr. A 1112, 369-374. doi: 10.1016/j.chroma.2005.12.056

Luan, F., Mosandl, A., Münch, A., and Wüst, M. (2005). Metabolism of geraniol in grape berry mesocarp of Vitis vinifera L. cv. Scheurebe: demonstration of stereoselective reduction, E/Z-isomerization, oxidation and glycosylation. Phytochemistry 66, 295-303. doi: 10.1016/j.phytochem.2004.12.017

Luan, F., and Wüst, M. (2002). Differential incorporation of 1-deoxy-Dxylulose into (3S)-linalool and geraniol in grape berry exocarp and mesocarp. Phytochemistry 60, 451-459. doi: 10.1016/S0031-9422(02)00147-4

Madeira, S. C., and Oliveira, A. L. (2004). Biclustering algorithms for biological data analysis: a survey. IEEE/ACM Trans. Comput. Biol. Bioinf. 1, 24-45. doi: 10.1109/TCBB.2004.2

Magnard, J. L., Roccia, A., Caissard, J. C., Vergne, P., Sun, P., Hecquet, R., et al. (2015). Plant volatiles. Biosynthesis of monoterpene scent compounds in roses. Science 349, 81-83. doi: 10.1126/science.aab0696

Malacarne, G., Costantini, L., Coller, E., Battilana, J., Velasco, R., Vrhovsek, U., et al. (2015). Regulation of flavonol content and composition in
$($ Syrah $\times$ Pinot Noir) mature grapes: integration of transcriptional profiling and metabolic quantitative trait locus analyses. J. Exp. Bot. 66, 4441-4453. doi: $10.1093 /$ jxb/erv243

Mannen, K., Matsumoto, T., Takahashi, S., Yamaguchi, Y., Tsukagoshi, M., Sano, R., et al. (2014). Coordinated transcriptional regulation of isopentenyl diphosphate biosynthetic pathway enzymes in plastids by phytochromeinteracting factor 5. Biochem. Biophys. Res. Commun. 443, 768-774. doi: 10.1016/j.bbrc.2013.12.040

Martin, D. M., Aubourg, S., Schouwey, M. B., Daviet, L., Schalk, M., Toub, O., et al. (2010). Functional annotation, genome organization and phylogeny of the grapevine (Vitis vinifera) terpene synthase gene family based on genome assembly, FLcDNA cloning, and enzyme assays. BMC Plant Biol. 10:226. doi: 10.1186/1471-2229-10-226

Martin, D. M., and Bohlmann, J. (2004). Identification of Vitis vinifera (-)- $\alpha$-terpineol synthase by in silico screening of full-length cDNA ESTs and functional characterization of recombinant terpene synthase. Phytochemistry 65, 1223-1229. doi: 10.1016/j.phytochem.2004 03.018

Martin, D. M., Chiang, A., Lund, S. T., and Bohlmann, J. (2012). Biosynthesis of wine aroma: transcript profiles of hydroxymethylbutenyl diphosphate reductase, geranyl diphosphate synthase, and linalool/nerolidol synthase parallel monoterpenol glycoside accumulation in Gewürztraminer grapes. Planta 236, 919-929. doi: 10.1007/s00425-012-1704-0

Matarese, F., Cuzzola, A., Scalabrelli, G., and D'Onofrio, C. (2014). Expression of terpene synthase genes associated with the formation of volatiles in different organs of Vitis vinifera. Phytochemistry 105, 12-24. doi: 10.1016/j.phytochem.2014.06.007

Matarese, F., Scalabrelli, G., and D'Onofrio, C. (2013). Analysis of the expression of terpene synthase genes in relation to aroma content in two aromatic Vitis vinifera varieties. Funct. Plant Biol. 40, 552-565. doi: 10.1071/FP12326

Mathieu, S., Wirth, J., Sauvage, F. X., Lepoutre, J. P., Baumes, R., and Gunata, Z. (2009). Biotransformation of $C_{13}$-norisoprenoids and monoterpenes by a cell suspension culture of cv. Gamay (Vitis vinifera). Plant Cell Tiss. Org. 97, 203-213. doi: 10.1007/s11240-009-9516-Z

Matus, J. T. (2016). Transcriptomic and metabolomic networks in the grape berry illustrate that it takes more than flavonoids to fight against ultraviolet radiation. Front. Plant Sci. 7:1337. doi: 10.3389/fpls.2016.01337

May, B., and Wüst, M. (2015). "Induction of de novo mono- and sesquiterpene biosynthesis by methyl jasmonate in grape berry exocarp," in Advances in Wine Research, ACS Symposium Series (Washington, DC), 1203, 191-201. doi: 10.1021/bk-2015-1203.ch012

Meesters, R. J., Duisken, M., and Hollender, J. (2007). Study on the cytochrome P450-mediated oxidative metabolism of the terpene alcohol linalool: indication of biological epoxidation. Xenobiotica 37, 604-617. doi: 10.3109/00498250701393191

Mendoza-Poudereux, I., Muñoz-Bertomeu, J., Arrillaga, I., and Segura, J. (2014). Deoxyxylulose 5-phosphate reductoisomerase is not a rate-determining enzyme for essential oil production in spike lavender. J. Plant Physiol. 171, 1564-1570. doi: 10.1016/j.jplph.2014.07.012

Nelson, D. R., Ming, R., Alam, M., and Schuler, M. A. (2008). Comparison of cytochrome P450 genes from six plant genomes. Trop. Plant Biol. 1, 216-235. doi: $10.1007 / \mathrm{s} 12042-008-9022-1$

Nieuwenhuizen, N. J., Chen, X., Wang, M. Y., Matich, A. J., Perez, R. L., Allan, A. C., et al. (2015). Natural variation in monoterpene synthesis in kiwifruit: transcriptional regulation of terpene synthases by NAC and ETHYLENEINSENSITIVE3-like transcription factors. Plant Physiol. 167, 1243-1258. doi: $10.1104 /$ pp.114.254367

Novikov, E., and Barillot, E. (2007). Software package for automatic microarray image analysis (MAIA). Bioinformatics 23, 639-640. doi: 10.1093/bioinformatics/btl644

Ong, P. K. C., and Acree, T. E. (1999). Similarities in the aroma chemistry of Gewürztraminer variety wines and lychee (Litchi chinesis Sonn.) fruit. J. Agric. Food Chem. 47, 665-670. doi: 10.1021/jf980452j

Palumbo, M. C., Zenoni, S., Fasoli, M., Massonnet, M., Farina, L., Castiglione, F., et al. (2014). Integrated network analysis identifies fight-club nodes as a class of hubs encompassing key putative switch genes that induce major transcriptome reprogramming during grapevine development. Plant Cell 26, 4617-4635. doi: 10.1105/tpc.114.133710 
Panighel, A., and Flamini, R. (2014). Applications of solid-phase microextraction and gas chromatography/mass spectrometry (SPME-GC/MS) in the study of grape and wine volatile compounds. Molecules 19, 21291-21309. doi: 10.3390/molecules191221291

Park, S. K., Morrison, J. C., Adams, D. O., and Noble, A. C. (1991). Distribution of free and glycosidically bound monoterpenes in the skin and mesocarp of Muscat of Alexandria grapes during development. J. Agric. Food Chem. 39, 514-518. doi: 10.1021/jf00003a017

Patra, B., Schluttenhofer, C., Wu, Y., Pattanaik, S., and Yuan, L. (2013). Transcriptional regulation of secondary metabolite biosynthesis in plants. Biochim. Biophys. Acta 1829, 1236-1247. doi: 10.1016/j.bbagrm.2013.09.006

Pauw, B., Hilliou, F. A., Martin, V. S., Chatel, G., de Wolf, C. J., Champion, A., et al. (2004). Zinc finger proteins act as transcriptional repressors of alkaloid biosynthesis genes in Catharanthus roseus. J. Biol. Chem. 279, 52940-52948. doi: 10.1074/jbc.M404391200

Piazzolla, F., Pati, S., Amodio, M. L., and Colelli, G. (2016). Effect of harvest time on table grape quality during on-vine storage. J. Sci. Food Agric. 96, 131-139. doi: 10.1002/jsfa.7072

Pokhilko, A., Bou-Torrent, J., Pulido, P., Rodríguez-Concepción, M., and Ebenhöh, O. (2015). Mathematical modelling of the diurnal regulation of the MEP pathway in Arabidopsis. New Phytol. 206, 1075-1085. doi: $10.1111 /$ nph.13258

Rapp, A. (1998). Volatile flavour of wine: correlation between instrumental analysis and sensory perception. Nahrung 42, 351-363. doi: 10.1002/(SICI)1521-3803(199812)42:063.3.CO;2-U

Reeves, P. H., Ellis, C. M., Ploense, S. E., Wu, M. F., Yadav, V., Tholl, D., et al. (2012). A regulatory network for coordinated flower maturation. PLoS Genet. 8:e1002506. doi: 10.1371/journal.pgen.1002506

Ribéreau-Gayon, P., Boidron, J. N., and Terrier, A. (1975). Aroma of Muscat grape varieties. J. Agric. Food Chem. 23, 1042-1047. doi: 10.1021/jf60202a050

Ribéreau-Gayon, P., Glories, Y., Maujean, A., and Dubourdieu, D. (2000). "Varietal aroma," in Handbook of Enology: The Chemistry of Wine Stabilization and Treatments (Chichester: John Wiley and Sons Ltd.), 205-230.

Robinson, A. L., Boss, P. K., Solomon, P. S., Trengove, R. D., Heymann, H., and Ebeler, S. E. (2014). Origins of grape and wine aroma. Part 1. Chemical components and viticultural impacts. Am. J. Enol. Vitic. 65, 1-24. doi: 10.5344/ajev.2013.12070

Rodríguez-Concepción, M. (2006). Early steps in isoprenoid biosynthesis: multilevel regulation of the supply of common precursors in plant cells. Phytochem. Rev. 5, 1-15. doi: 10.1007/s11101-005-3130-4

Rodríguez-Concepción, M., Ahumada, I., Diez-Juez, E., Sauret-Güeto, S., Lois, L. M., Gallego, F., et al. (2001). 1-Deoxy-D-xylulose 5-phosphate reductoisomerase and plastid isoprenoid biosynthesis during tomato fruit ripening. Plant J. 27, 213-222. doi: 10.1046/j.1365-313x.2001.01089.x

Rodríguez-Concepción, M., and Boronat, A. (2015). Breaking new ground in the regulation of the early steps of plant isoprenoid biosynthesis. Curr. Opin. Plant Biol. 25, 17-22. doi: 10.1016/j.pbi.2015.04.001

Rotter, A., Camps, C., Lohse, M., Kappel, C., Pilati, S., Hren, M., et al. (2009). Gene expression profiling in susceptible interaction of grapevine with its fungal pathogen Eutypa lata: extending MapMan ontology for grapevine. BMC Plant Biol. 9:104. doi: 10.1186/1471-2229-9-104

Ruiz-García, L., Hellín, P., Flores, P., and Fenoll, J. (2014). Prediction of Muscat aroma in table grape by analysis of rose oxide. Food Chem. 154, 151-157. doi: 10.1016/j.foodchem.2014.01.005

Ruwe, H., Kupsch, C., Teubner, M., and Schmitz-Linneweber, C. (2011). The RNA-recognition motif in chloroplasts. J. Plant Physiol. 168: 1361-1371. doi: 10.1016/j.jplph.2011.01.012

Sánchez Palomo, E., Pérez-Coello, M. S., Díaz-Maroto, M. C., González Viñas, M. A., and Cabezudo, M. D. (2006). Contribution of free and glycosidically-bound volatile compounds to the aroma of muscat "à petit grains" wines and effect of skin contact. Food Chem. 95, 279-289. doi: 10.1016/j.foodchem.2005.01.012

Sasaki, K., Takase, H., Matsuyama, S., Kobayashi, H., Matsuo, H., Ikoma, G., et al. (2016). Effect of light exposure on linalool biosynthesis and accumulation in grape berries. Biosci. Biotechnol. Biochem. 80, 2376-2382. doi: 10.1080/09168451.2016.1217148

Savoi, S., Wong, D. C. J., Arapitsas, P., Miculan, M., Bucchetti, B., Peterlunger, E., et al. (2016). Transcriptome and metabolite profiling reveals that prolonged drought modulates the phenylpropanoid and terpenoid pathway in white grapes (Vitis vinifera L.). BMC Plant Biol. 16:67. doi: 10.1186/s12870-016-0760-1

Schwab, W. (2003). Metabolome diversity: too few genes, too many metabolites? Phytochemistry 62, 837-849. doi: 10.1016/S0031-9422(02)00723-9

Schwab, W., and Wüst, M. (2015). Understanding the constitutive and induced biosynthesis of mono- and sesquiterpenes in grapes (Vitis vinifera) - A key to unlocking the biochemical secrets of unique grape aroma profiles. J. Agric. Food Chem. 63, 10591-10603. doi: 10.1021/acs.jafc.5b04398

Shen, Y., Wei, W., and Zhou, D. X. (2015). Histone acetylation enzymes coordinate metabolism and gene expression. Trends Plant. Sci. 20, 614-621. doi: 10.1016/j.tplants.2015.07.005

Sibéril, Y., Benhamron, S., Memelink, J., Giglioli-Guivarc'h, N., Thiersault, M., Boisson, B., et al. (2001). Catharanthus roseus G-box binding factors 1 and 2 act as repressors of strictosidine synthase gene expression in cell cultures. Plant Mol Biol. 45, 477-488. doi: 10.1023/A:1010650906695

Smyth, G. K. (2004). Linear models and empirical bayes methods for assessing differential expression in microarray experiments. Stat. Appl. Genet. Mol. Biol. 3:3. doi: 10.2202/1544-6115.1027

Sun, P., Schuurink, R. C., Caissard, J. C., Hugueney, P., and Baudino, S. (2016). My way: noncanonical biosynthesis pathways for plant volatiles. Trends Plant Sci. 21, 884-894. doi: 10.1016/j.tplants.2016.07.007

Suzuki, M., Nakabayashi, R., Ogata, Y., Sakurai, N., Tokimatsu, T., Goto, S., et al. (2015). Multiomics in grape berry skin revealed specific induction of the stilbene synthetic pathway by ultraviolet-C irradiation. Plant Physiol. 168, 47-59. doi: 10.1104/pp.114.254375

Suzuki, R., and Shimodaira, H. (2006). Pvclust: an R package for assessing the uncertainty in hierarchical clustering. Bioinformatics 22, 1540-1542. doi: 10.1093/bioinformatics/btl117

Tholl, D., Kish, C. M., Orlova, I., Sherman, D., Gershenzon, J., Pichersky, E., et al. (2004). Formation of monoterpenes in Antirrhinum majus and Clarkia breweri flowers involves heterodimeric geranyl diphosphate synthases. Plant Cell 16, 977-992. doi: 10.1105/tpc.020156

Ting, H. M., Delatte, T. L., Kolkman, P., Misas-Villamil, J. C., van der Hoorn, R. A., Bouwmeester, H. J., et al. (2015). SNARE-RNAi results in higher terpene emission from ectopically expressed caryophyllene synthase in Nicotiana benthamiana. Mol. Plant 8, 454-466. doi: 10.1016/j.molp.2015.01.006

Toledo-Ortiz, G., Johansson, H., Lee, K. P., Bou-Torrent, J., Stewart, K., Steel, G., et al. (2014). The HY5-PIF regulatory module coordinates light and temperature control of photosynthetic gene transcription. PLoS Genet. 10:e1004416. doi: 10.1371/journal.pgen.1004416

Untergasser, A., Cutcutache, I., Koressaar, T., Ye, J., Faircloth, B. C., Remm, M., et al. (2012). Primer3-new capabilities and interfaces. Nucleic Acids Res. 40:e115. doi: 10.1093/nar/gks596

Velasco, R., Zharkikh, A., Troggio, M., Cartwright, D. A., Cestaro, A., Pruss, D., et al. (2007). A high quality draft consensus sequence of the genome of a heterozygous grapevine variety. PLoS ONE 2:e1326. doi: 10.1371/journal.pone.0001326

Vilanova, M., Genisheva, Z., Bescansa, L., Masa, A., and Oliveira, J. M. (2012). Changes in free and bound fractions of aroma compounds of four Vitis vinifera cultivars at the last ripening stages. Phytochemistry 74, 196-205. doi: 10.1016/j.phytochem.2011.10.004

Vom Endt, D., Kijne, J. W., and Memelink, J. (2002). Transcription factors controlling plant secondary metabolism: what regulates the regulators? Phytochemistry 61, 107-114. doi: 10.1016/S0031-9422(02)00185-1

Vranová, E., Coman, D., and Gruissem, W. (2012). Structure and dynamics of the isoprenoid pathway network. Mol. Plant 5, 318-333. doi: 10.1093/mp/sss015

Wang, Q., Reddy, V. A., Panicker, D., Mao, H. Z., Kumar, N., Rajan, C., et al. (2016). Metabolic engineering of terpene biosynthesis in plants using a trichomespecific transcription factor MsYABBY5 from spearmint (Mentha spicata). Plant Biotechnol J. 14, 1619-1632. doi: 10.1111/pbi.12525

Wen, Y. Q., Zhong, G. Y., Gao, Y., Lan, Y. B., Duan, C. Q., and Pan, Q. H. (2015). Using the combined analysis of transcripts and metabolites to propose key genes for differential terpene accumulation across two regions. BMC Plant Biol. 15:240. doi: 10.1186/s12870-015-0631-1

Williams, P. J., Sefton, M. A., and Wilson, B. (1989). "Nonvolatile conjugates of secondary metabolites as precursors of varietal grape flavor components," in Flavor Chemistry Trends and Developments, eds R. Teranishi, R. G. Buttery, and F. Shahidi (Washington, DC: American Chemical Society), 35-48. 
Wilson, B., Strauss, C. R., and Williams, P. J. (1986). The distribution of free and glycosydically-bound monoterpenes among skin, juice, and pulp fractions of some white grape varieties. Am. J. Enol. Vitic. 37, 107-111

Wong, D. C. J., Schlechter, R., Vannozzi, A., Höll, J., Hmmam, I., Bogs, J., et al. (2016). A systems-oriented analysis of the grapevine R2R3-MYB transcription factor family uncovers new insights into the regulation of stilbene accumulation. DNA Res. 23, 451-466. doi: 10.1093/dnares/dsw028

Yuan, J. S., Galbraith, D. W., Dai, S. Y., Griffin, P., and Stewart, C. N. Jr. (2008). Plant systems biology comes of age. Trends Plant Sci. 13, 165-171. doi: $10.1016 /$ j.tplants.2008.02.003

Zamboni, A., Di Carli, M., Guzzo, F., Stocchero, M., Zenoni, S., Ferrarini, A., et al. (2010). Identification of putative stage-specific grapevine berry biomarkers and omics data integration into networks. Plant Physiol. 154, 1439-1459. doi: 10.1104/pp.110.160275

Zhang, H., Fan, P., Liu, C., Wu, B., Li, S., and Liang, Z. (2014). Sunlight exclusion from Muscat grape alters volatile profiles during berry development. Food Chem. 164, 242-250. doi: 10.1016/j.foodchem.2014.05.012

Zhou, F., Sun, T. H., Zhao, L., Pan, X. W., and Lu, S. (2015). The bZIP transcription factor HY5 interacts with the promoter of the monoterpene synthase gene QH6 in modulating its rhythmic expression. Front. Plant Sci. 6:304. doi: $10.3389 /$ fpls.2015.00304

Zhu, B. Q., Cai, J., Wang, Z. Q., Xu, X. Q., Duan, C. Q., and Pan, Q. H. (2014). Identification of a plastid-localized bifunctional nerolidol/linalool synthase in relation to linalool biosynthesis in young grape berries. Int. J. Mol. Sci. 15, 21992-22010. doi: 10.3390/ijms1512 21992

Conflict of Interest Statement: The authors declare that the research was conducted in the absence of any commercial or financial relationships that could be construed as a potential conflict of interest.

Copyright (c) 2017 Costantini, Kappel, Trenti, Battilana, Emanuelli, Sordo, Moretto, Camps, Larcher, Delrot and Grando. This is an open-access article distributed under the terms of the Creative Commons Attribution License (CC BY). The use, distribution or reproduction in other forums is permitted, provided the original author(s) or licensor are credited and that the original publication in this journal is cited, in accordance with accepted academic practice. No use, distribution or reproduction is permitted which does not comply with these terms. 